\title{
Observations of the SW Sextantis Star UU Aquarii
}

\author{
D. W. Hoard ${ }^{1}$ M. D. Still, ${ }^{2}$ Paula Szkody, ${ }^{1}$ Robert Connon Smith,${ }^{3}$ and D. A. H. Buckley
}

\begin{abstract}

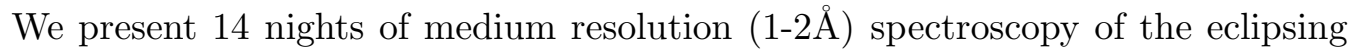
cataclysmic variable UU Aquarii obtained during a high accretion state in 1995 August-October. UU Aqr appears to be an SW Sextantis star, as noted by Baptista, Steiner, \& Horne (1996), and we discuss its spectroscopic behavior in the context of the SW Sex phenomenon. Emission line equivalent width curves, Doppler tomography, and line profile simulation provide evidence for the presence of a bright spot at the impact site of the accretion stream with the edge of the disk, and a non-axisymmetric, vertically- and azimuthally-extended absorbing structure in the disk. The absorption has maximum depth in the emission lines around orbital phase 0.8 , but is present from $\phi \approx 0.4$ to $\phi \approx 0.95$. An origin is explored for this absorbing structure (as well as the other spectroscopic behavior of UU Aqr) in terms of the explosive impact of the accretion stream with the disk.
\end{abstract}

Subject headings: accretion, accretion disks — novae, cataclysmic variables — stars: individual (UU Aquarii)

\section{Introduction}

Cataclysmic variables $(\mathrm{CVs})$ are short period $\left(\mathrm{P}_{\text {orb }}\right.$ typically $\left.\lesssim 1 \mathrm{~d}\right)$, semi-detached binary systems in which matter is transferred through the $L_{1}$ point from a Roche-lobe-filling, lower main sequence dwarf (the secondary star), into an accretion disk around the white dwarf (WD) primary star. In most nonmagnetic CVs, energy output from the disk dominates the optical-X-ray luminosity; hence, the properties of the disk (which are primarily governed by the mass transfer rate $\dot{M}$ in the case of an optically thick disk) determine most of the observed properties of the

\footnotetext{
${ }^{1}$ Department of Astronomy, University of Washington, Box 351580, Seattle, WA 98195-1580, USA; email: hoard@astro.washington.edu, szkody@astro.washington.edu

${ }^{2}$ Physics \& Astronomy, University of St. Andrews, North Haugh, St. Andrews, Fife KY16 9SS, UK; email: martin.still@st-and.ac.uk

${ }^{3}$ Astronomy Centre, University of Sussex, Brighton BN1 9QJ, UK; email: rcs@star.maps.susx.ac.uk

${ }^{4}$ South African Astronomical Observatory, PO Box 9, Observatory 7935, Cape Town, South Africa; email: dibnob@saao.ac.za
} 
entire system. UU Aquarii $(V \approx 13.5)$ was identified as a variable star early in this century (Beljawsky 1926). As observations of UU Aqr have been accumulated, its classification has evolved from a long-period semi-regular variable (Payne-Gaposchkin 1952), to a low $\dot{M}$, optically thin disk, dwarf nova type CV (Volkov, Shugarov, \& Seregina 1986), to a high $\dot{M}$, optically thick disk, novalike CV (Diaz \& Steiner 1991, henceforth DS91; Baptista, Steiner, \& Cieslinski 1994, henceforth BSC94).

Volkov et al. (1986) and Goldader \& Garnavich (1989) observed deep $(\approx 1.5$ mag) eclipses in UU Aqr, and established an orbital period of $\mathrm{P}_{\text {orb }}=0.163579089(61) \mathrm{d}(\approx 3.93 \mathrm{~h})$. This places UU Aqr in the 3-4 hr period range just above the 2-3 hr CV period gap (thought to be due to the transition from orbital angular momentum loss primarily via magnetic braking for $\mathrm{P}>3 \mathrm{hr}$ to loss due to gravitational radiation for $\mathrm{P}<2 \mathrm{hr}$; King 1988). Among novalike CVs, the 3-4 hr period range is occupied almost solely by the group of systems known as the $S W$ Sextantis stars (e.g., Thorstensen et al. 1991), which are characterized by high inclination, single-peaked optical emission lines (in contrast to the double-peaked lines expected in high inclination disk systems), orbital-phase-dependent absorption in the Balmer lines, and a phase offset in the emission line radial velocity curves (implying non-uniform emission from the disk). As a result of their multiwavelength eclipse mapping study, Baptista, Steiner, \& Horne (1996; henceforth BSH96) conclude that UU Aqr is most likely an SW Sex star (a conclusion that we share based on the results presented here - see $\S 4.4)$.

BSC94 obtained photometric data of UU Aqr on 28 nights during 1988-1992. They discovered that this system, like many other novalike CVs (Dhillon 1996), exhibits occasional transitions from high to low brightness states. The amplitude of the state change in UU Aqr, however, is only $\approx 0.2-0.3$ mag, which is small compared to other novalike CVs (e.g., BH Lyncis: $\Delta$ mag $\approx 1.5-2.0$, Hoard \& Szkody 1997; DW Ursae Majoris: $\Delta$ mag $\approx 2-3$, Dhillon, Jones, \& Marsh 1994). The shape of the eclipse profile in UU Aqr does change significantly from the high to low states, and BSC94 concluded that the main difference between the two states is the presence of a bright spot on the outer edge of the disk in the high state.

Both Haefner (1989) and DS91 determined radial velocity curves for the $\mathrm{H} \alpha$ line in UU Aqr; both noted the strongly asymmetric, phase-dependent profile of the line. Haefner (1989) found a velocity semi-amplitude of $160 \mathrm{~km} \mathrm{~s}^{-1}$ from the wings of the $\mathrm{H} \alpha$ line, while DS91 obtained 120 $\mathrm{km} \mathrm{s}^{-1}$ using the same method. DS91 determined system parameters of $q=\left(\mathrm{M}_{2} / \mathrm{M}_{w d}=\right) 0.4$ with $\mathrm{M}_{w d}=0.9 \mathrm{M}_{\odot}$. More recently, the detailed eclipse-mapping study of UU Aqr by BSC94 and BSH96 provided a photometric model that requires $K_{w d} \approx 84 \mathrm{~km} \mathrm{~s}^{-1}$, substantially smaller than estimates made from the $\mathrm{H} \alpha$ emission line. (This is not surprising as low excitation emission lines, which form primarily in the outer parts of the disk, cannot be expected to accurately map the orbital motion of the WD; e.g., Still, Dhillon, \& Jones 1995, Shafter, Hessman, \& Zhang 1988.) BSC94 derive system parameters of $q=0.30$ with $\mathrm{M}_{w d}=0.67 \mathrm{M}_{\odot}$ and an inclination $i=78^{\circ}$.

We present here the results of an extensive spectroscopic monitoring campaign on UU Aqr. 
Previously published time-resolved spectra (DS91) were obtained during a low state, while our spectra explore the high state of the system (see $\$ 3.3$ ). In addition, the spectra of DS91 covered only $200 \AA$ around $\mathrm{H} \alpha$, whereas our spectra span $\mathrm{H} \beta$ to $\mathrm{H} \gamma$ on all nights, with additional coverage from $\mathrm{H} \alpha$ to $\mathrm{He}$ I $\lambda 5876$ on one night. Our results include a line profile simulation that reproduces the gross characteristics of the orbital-phase-resolved emission line behavior of UU Aqr in terms of several discrete emission and absorption components (\$4.1), Doppler tomography resolved over half an orbit of the CV (\$4.2), and a qualitative model for the system (\$4.3).

\section{Observations}

We observed UU Aqr using the 1.9m Radcliffe Telescope at the South African Astronomical Observatory (SAAO) on 1995 August 1-8 UT, 1995 August 18-20 UT, and 1995 September 19-26 UT. The observations were made using the two-channel Reticon Photon Counting System (RPCS; Menzies \& Glass 1996) with a 1200 line $\mathrm{mm}^{-1}$ grating (giving a resolution of $1.8 \AA$ ). Exposures were $90 \mathrm{~s}$ with a total time per spectrum of $\approx 97 \mathrm{~s}$; a total of 1492 spectra were obtained. Sky was recorded in one channel of the RPCS and sky+object in the other channel. Following normalization via bias-subtraction and division by a tungsten lamp flat-field, the spectra were extracted by simply subtracting the sky channel from the sky+object channel. The spectra were wavelength-calibrated by comparison to spectra of a $\mathrm{Cu}$-Ar arc lamp standard; the usable wavelength range was $4250-5000 \AA$. They were flux-calibrated via observations of the standard stars L1788 and L7379 (Hamuy et al. 1994). In order to increase the signal-to-noise, the reduced RPCS spectra were averaged into phase bins of width $\Delta \phi=0.04$ in blocks of 2 consecutive nights (see Table 1), using the photometric ephemeris of BSC94 (we will refer to these as the "binned RPCS data sets"). We also constructed sets of phase-binned $(\Delta \phi=0.04)$ spectra using $\approx 1450$ of the total set of RPCS spectra (some of the RPCS spectra were rejected because they were extremely weak, possibly due to slit losses; we will refer to this as the "combined RPCS data set").

We also obtained spectra of UU Aqr on 1995 October 12-13 UT using the Double Imaging Spectrograph (DIS) on the Apache Point Observatory 3.5m telescope (e.g., see Hoard \& Szkody 1997). Exposure times were $300 \mathrm{~s}$ with a total time of $\approx 381 \mathrm{~s}$ per spectrum; a total of 65

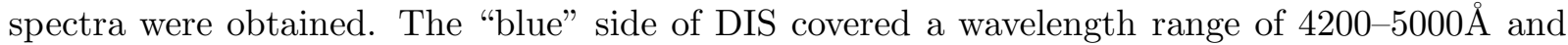
the "red" side covered $5800-6800 \AA$, with an overall resolution of $\approx 1.5-2 \AA$. The spectra were extracted and calibrated using standard IRAF routines (Massey, Valdes, \& Barnes 1992); they were wavelength-calibrated via comparison with a He-Ne arc lamp standard. Fine corrections to the wavelength calibration were made by examining the positions of night sky lines in the spectra. Both the blue and red spectra were flux-calibrated using spectra of the standard stars Feige 110 and $\mathrm{BD}+28^{\circ} 4211$ (Massey et al. 1988).

The spectroscopic observations of UU Aqr are summarized in Table 1. 


\section{Results}

\subsection{The Average Spectrum}

The average spectrum of UU Aqr, outside orbital phases 0.9 to 0.1 (the eclipse) and uncorrected for any orbital motion, is shown in Figure 11. Each blue spectrum from the binned RPCS data sets listed in Table 1 is plotted in the figure. The spectrum shows prominent Balmer emission lines and several weaker He I emission lines $(\lambda 4471, \lambda 4921, \lambda 5015, \lambda 5876, \lambda 6678)$. The He II $\lambda 4686$ emission line is weak and blended with a C III/N III emission complex of similar strength. All of the emission lines are single-peaked (this is true for the individual spectra as well for example, see Fig. 10 - so this is not just an effect of smearing of the line profiles in the average spectrum). The blue spectrum is quite constant over the $\approx 3$ month span of the observations. The continuum from $4200 \AA$ to $6800 \AA$ follows a power law, $F_{\lambda} \propto \lambda^{-\alpha}$ with $\alpha \approx 1.7$. This is somewhat smaller than the power law index $\alpha=2.3$ expected from both theoretical calculations in which all disk elements radiate as blackbodies (Pringle 1981) and observations of dwarf novae at outburst (Szkody 1985). A relatively flat spectral energy distribution is considered typical of novalike CVs (Oke \& Wade 1982), and may be related to departures of the disk structure from simple theoretical models and/or self-eclipse of the inner disk in high inclination systems (e.g., Rutten, van Paradijs, \& Tinbergen 1992).

\subsection{Radial Velocities}

Radial velocities were measured from the wings of the Balmer emission lines in all of the binned RPCS data sets, and from the Balmer and helium lines in each of the unbinned DIS spectra, using the double Gaussian fitting technique described by Shafter, Szkody, \& Thorstensen (1986) and Schneider \& Young (1980). Before applying this technique to each line, a section of the spectrum around the wavelength of the line center $\left( \pm 3000 \mathrm{~km} \mathrm{~s}^{-1}\right)$ was converted to a uniform (i.e., linear) velocity scale with a step-size of the local velocity dispersion,

$$
(c \Delta \lambda) / \lambda_{0} \mathrm{~km} \mathrm{~s}^{-1},
$$

where $c$ is the speed of light $\left(\mathrm{km} \mathrm{s}^{-1}\right), \lambda_{o}$ is the wavelength of the line center $(\AA)$, and $\Delta \lambda$ is the wavelength dispersion $(\approx 0.8 \AA$ for the RPCS spectra, $\approx 1.3 \AA$ for the red DIS spectra, and $\approx 1.6 \AA$ for the blue DIS spectra). For the Balmer and He I $\lambda 5876$ lines, the FWHM of the Gaussians was set at twice the local velocity resolution of the spectra for each line $\left(\mathrm{FWHM} \approx 160 \mathrm{~km} \mathrm{~s}^{-1}\right.$ at $\mathrm{H} \beta$ to $180 \mathrm{~km} \mathrm{~s}^{-1}$ at $\mathrm{H} \gamma$ in the RPCS spectra; FWHM $\approx 120 \mathrm{~km} \mathrm{~s}^{-1}$ at $\mathrm{H} \alpha$ to $230 \mathrm{~km} \mathrm{~s}^{-1}$ at $\mathrm{H} \gamma$ in the DIS spectra). The Gaussian separation $a$ was varied in steps of $100 \mathrm{~km} \mathrm{~s}^{-1}$ from $500 \mathrm{~km} \mathrm{~s}^{-1}$

to $2500 \mathrm{~km} \mathrm{~s}^{-1}$. In order to obtain usable velocities from the weaker He I $\lambda 4471$ and He II $\lambda 4686$ lines (in the DIS spectra), the FWHM of the Gaussians was increased to $400 \mathrm{~km} \mathrm{~s}^{-1}$.

The equation

$$
V(\phi, a)=\gamma(a)-K(a) \sin \left[2 \pi\left(\phi-\phi_{0}(a)\right)\right]
$$


was fitted to the velocity data obtained from the optimum value of the Gaussian separation for each line. Because we are comparing spectra obtained at different times, the velocities from each data set were shifted by the appropriate heliocentric correction prior to fitting. Representative samples of the diagnostic diagrams (from the DIS data) used to determine the optimum Gaussian separation are shown in Figure 2. For the Balmer lines, the diagrams are well-behaved and we chose $a=1700 \mathrm{~km} \mathrm{~s}^{-1}$. The diagrams for the helium lines, on the other hand, are rather more uncertain. All three He lines display a trough in the fractional uncertainty of the velocity semi-amplitude, $\sigma_{K} / K$, between $a=1100 \mathrm{~km} \mathrm{~s}^{-1}$ and $a=1800 \mathrm{~km} \mathrm{~s}^{-1}$, indicating that this range of Gaussian separations best samples the line wings away from the highly variable line cores but before the adjacent continuum contaminates the wings significantly. However, the values of the system parameters (especially $K$ ) still fluctuate significantly over this range of $a$. The He I $\lambda 5876$ line is the best-behaved of the He lines, and we have used $a=1700 \mathrm{~km} \mathrm{~s}^{-1}$ for this line also, based on the overall shapes of its $\sigma_{K} / K, K$, and $\sigma_{\text {total }}$ curves, which are similar to those of the Balmer lines. The values (and $1 \sigma$ uncertainies determined from Monte Carlo simulations) of the UU Aqr velocity parameters for the Balmer and He I $\lambda 5876$ lines are listed in Table 2 for each of the data sets.

For He I $\lambda 4471$ and He II, the velocity parameter values between $a=1100 \mathrm{~km} \mathrm{~s}^{-1}$ and $a=1800 \mathrm{~km} \mathrm{~s}^{-1}$ (where $\sigma_{K} / K$ is smallest) oscillate around a roughly flat mean trend, which makes it difficult to pick an optimum Gaussian separation. Instead, we have computed a weighted average of the parameter values in this range of $a$ using

$$
\overline{x_{i}}=\frac{\Sigma\left(x_{i} / \sigma_{i}^{2}\right)}{\Sigma\left(1 / \sigma_{i}^{2}\right)},
$$

where $\sigma_{i}$ is the uncertainty in parameter $x_{i}$ at a given $a$ as determined from a Monte Carlo simulation. This provides a representative velocity solution, rather than relying on any one of the individual solutions (which might lie above or below the general trend). The average parameters for these two lines are also listed in Table 2. The Balmer, He I $\lambda 4471$ and $\lambda 5876$, and He II $\lambda 4686$ radial velocity curves obtained from the DIS spectra are shown in Figure 3, along with the best fit sine functions. For the He I $\lambda 4471$ and He II lines, we have plotted the radial velocities for $a=1700 \mathrm{~km} \mathrm{~s}^{-1}$ with the sine function produced by the average velocity parameters. The RPCS radial velocity curves for $\mathrm{H} \beta$ and $\mathrm{H} \gamma$ do not differ significantly from the DIS curves, so are not plotted. The He lines in the RPCS data sets were generally too weak to yield reliable wing velocity curves.

All of the lines have positive phase offsets $\left(\phi_{0}\right)$ corresponding to a delay of the red-to-blue velocity crossing of the emission lines relative to photometric phase 0.0 . The phase offsets range from $\approx 0.1-0.2$ in the Balmer and He I lines, to $\approx 0.3$ in the He II line (again, however, we cannot rule out the possibility of a skewed result owing to blending of the He II line). This implies that the main source of emission is off-center in the disk. 
If we consider the systemic velocities $(\gamma)$ as a function of time, then the $\mathrm{H} \beta$ and $\mathrm{H} \gamma$ lines display similar behavior. In both lines, the gamma velocity decreases from data set \#1 to \#2. In $\mathrm{H} \beta$ this decrease continues through data set $\# 3$, then $\gamma$ has its maximum value in data set \#4; in $\mathrm{H} \gamma$ the maximum value is reached in data set \#3. The value of $\gamma$ then decreases following the maximum for each line, until a minimum value $(\gamma<0$ for $\mathrm{H} \beta$ and several of the other lines) is reached in data set \#7. This pattern does not appear to correlate with the smaller ( $\lesssim \pm 2 \sigma)$, somewhat more random fluctuations of the other velocity parameters $\left(K\right.$ and $\left.\phi_{0}\right)$. This suggests that the systemic velocity is influenced by a separate emission region than the velocity semi-amplitude and phase offset. For example, the presence of an emission component from a (non-rotating or only slightly rotating) disk wind could systematically skew the zero level of the radial velocities without overtly disrupting the rotational behavior of underlying emission from the disk. Variability of the flux of emitting material in this wind might then account for the changing $\gamma$ values in UU Aqr.

The semi-amplitude of the $\mathrm{H} \beta$ radial velocity ranges from $K=77-117 \mathrm{~km} \mathrm{~s}^{-1}$, with the best-determined value $\left(\sigma_{K} / K=0.05\right)$ from the DIS data, $K=115 \pm 6 \mathrm{~km} \mathrm{~s}^{-1}$. The variability of the $K$ value may be related to small changes in the location and/or structure of the off-center emitting region that dominates the Balmer lines. For the $\mathrm{H} \alpha$ line, the DIS spectra give $151 \pm 8$ $\mathrm{km} \mathrm{s}^{-1}$. This is slightly smaller than the $\mathrm{H} \alpha$ wing velocity $K \sim 160 \mathrm{~km} \mathrm{~s}^{-1}$ measured by Haefner (1989), but larger than that found by DS91, $K=121 \pm 7 \mathrm{~km} \mathrm{~s}^{-1}$. In general, the Balmer lines and (especially) the He II line have velocity semi-amplitudes larger than the nominal WD orbital velocity predicted by the photometric model of BSC94, $K_{w d}=84 \pm 26 \mathrm{~km} \mathrm{~s}^{-1}$, although many of the $\mathrm{H} \beta$ and $\mathrm{H} \gamma$ (but not $\mathrm{H} \alpha$ or $\mathrm{He}$ II) velocities are within $\approx 1 \sigma$ of the BSC94 value. The He II line is often thought to originate in the hotter region close to the WD and, hence, to be a good indicator of the WD orbital velocity. However, both its large difference from the independently determined value of BSC94 and its large phase offset argue against adopting our value for $K_{H e I I}$ as $K_{w d}$. Thus, we will refrain from computing additional system parameters and will instead accept those determined for UU Aqr by BSC94 from their multicolor eclipse mapping investigation (i.e., $q=0.30 \pm 0.07, \mathrm{M}_{w d}=0.67 \pm 0.14 \mathrm{M}_{\odot}, \mathrm{M}_{2}=0.20 \pm 0.07 \mathrm{M}_{\odot}$, and $i=78 \pm 2^{\circ}$ ). We note for the sake of completeness that the effect on the derived system parameters of adopting a larger WD orbital velocity would be to increase $q$ and decrease $i$ (e.g., Garnavich et al. 1990).

\subsection{Equivalent Widths}

We measured equivalent widths (EWs) for all 65 of the DIS spectra and for all of the spectra from the binned RPCS data sets. The EW curves for the DIS spectra are shown in Figure $\mathbb{1}$; the curves for the RPCS spectra do not differ in any significant way from the DIS curves, so are not shown. The mean EWs and $1 \sigma$ uncertainties outside of eclipse $(0.1 \leq \phi \leq 0.9)$ for the $\mathrm{H} \beta, \mathrm{H} \gamma$, He I $\lambda 4471$, and He II $\lambda 4686$ emission lines of UU Aqr are listed in Table 3 for each of the binned data sets. Note that the He II line is blended with the adjacent C III/N III emission complex and 
the EW measures the flux from both. The mean EWs of all of these lines were essentially constant from 1995 August to 1995 October. We note, however, that data set \#7 (which has the smallest emission line systemic velocities - see Table 2) has the smallest mean Balmer and He I EWs and the largest mean He II EW. This might be explained if the Balmer and He I emission contains a variable wind emission component that produces a positive systemic velocity when present (as discussed in $(3.2$ ), but is absent or reduced in amplitude in data set \#7.

Outside of eclipse, the EWs go through roughly sinusoidal modulations on the orbital period, with minima at $\phi \approx 0.7-0.8$. This modulation is most apparent in the $\mathrm{H} \alpha$ line, where it has a full amplitude of $\approx 15 \AA$ (see top right panel of Fig. 1 ). This modulation is not seen in broadband (i.e., continuum) light curves of UU Aqr (see Fig. 5 and text below), indicating that it is due to variability in the emission lines. In the novalike CVs BH Lyn (Hoard \& Szkody 1997) and PG 0859+415 (Hoard \& Szkody 1996a), similar modulation of the EWs was interpreted as due to absorption in a vertically extended "bulge" on the edge of the disk. The presence of such bulges in the disk edge is predicted at phases of $0.2,0.5$, and/or 0.8 by numerical simulations of accretion stream-disk interaction (e.g., Hirose, Osaki, \& Mineshige 1991; Meglicki, Wickramasinghe, \& Bicknell 1993). Absorption by a bulge at $\phi \approx 0.8$ could explain the UU Aqr EW modulation.

The most obvious feature of the EW curves is the presence of a large peak around $\phi \approx 0.0$ in all of them. The height of this peak varies from curve to curve; among the Balmer series in particular, its amplitude is largest for $\mathrm{H} \alpha(\approx 55 \AA)$ and smallest for $\mathrm{H} \gamma(\approx 20 \AA)$. The eclipse peaks reach maximum amplitude at $\phi=0.0$ but are asymmetrically shaped, with a steep rise before $\phi=0.0(\Delta \phi=0.03-0.05)$ and a longer decline $(\Delta \phi=0.05-0.10)$. DS91 reported no change in the emission line EWs during eclipse, but their spectra were obtained in 1988 October, when BSC94 determined that UU Aqr was in a low accretion state. Yet, the narrowband $(50 \AA) \mathrm{H} \alpha$ light curve of UU Aqr obtained by BSH96 during another low state (in 1992) is uneclipsed relative to light curves in both the broadband $R$ and adjacent narrowband $\lambda 6700$ filters. This would produce a peak in the $\mathrm{H} \alpha \mathrm{EW}$ as seen in our high state data. The lack of eclipse in the emission lines implies that there is an extended source of emission that remains partially visible during eclipse; however, the presence of such a region does not appear to be strongly tied to the brightness state in UU Aqr.

We acquired CCD photometry of UU Aqr at SAAO on the nights of 1995 August 01-02 and 06-07 UT, concurrent with binned RPCS data sets \#1 and \#2 (see Table 1). A total of 570 measurements were obtained, with typical time resolution of $\approx 69 \mathrm{~s}$, and calibrated to Strömgren b magnitudes via comparison to observations of E-region standards (e.g., Cousins 1987) obtained under photometric conditions. The eclipse profile in the resultant light curve of UU Aqr (shown in Fig. 5) is most similar in shape to that of the high state light curves of BSC94 (compare to their Fig. 5). The small differences in the profile from orbit to orbit immediately before and after eclipse are consistent with those seen by BSC94. The asymmetry of the EW peaks is reflected 
in the shape of the high state eclipse light curve, which shows a steep, smooth ingress to the eclipse minimum over $\Delta \phi \approx 0.04$, followed by a longer egress, $\Delta \phi \approx 0.1$, back to the quiescent level. The eclipse egress has a sharp shoulder $\Delta \phi \approx 0.04$ after the minimum. BSC94 interpreted the asymmetry of the eclipse as an effect of the bright spot, which must have a large azimuthal extent on the edge of the disk. The projected surface area of the spot along the line-of-sight to the system decreases as the orbital phase approaches $\phi=0.0$, leading to a rapid spot eclipse ingress. After the eclipse minimum, the projected spot area along the line-of-sight increases as $\phi$ increases, leading to a longer egress from the eclipse of the spot (also see Rutten et al. 1992).

In addition, the light curve of UU Aqr during 1995 from the Indiana Automated CCD Photometric Telescope ("Roboscope;" e.g., Honeycutt \& Turner 1992, Honeycutt et al. 1994) shows that the system was bright $(V=13.5 \pm 0.1)$ during our spectroscopic observations (Robertson 1997). As noted in $\S$ 团, the difference in brightness between high and low states of UU Aqr is small, but the eclipse profile shapes in the two states show significant differences; hence, the latter is a more precise diagnostic of the accretion state in UU Aqr than the former. Based on the comparison of the system brightness, EW behavior, and eclipse profile to previously published observations, we conclude that UU Aqr was in a high state during our observations.

\subsection{Doppler Tomography}

We used the Fourier-filtered back-projection algorithm described by Horne (1991) and others (e.g., Marsh \& Horne 1988; Kaitchuck et al. 1994) to produce Doppler tomograms of the velocity distribution of emitting material in UU Aqr. Figures $6 \mathrm{a}-\mathrm{f}$ show the $\mathrm{H} \beta$ tomograms for the six binned RPCS data sets; Figure $6 \mathrm{~g}$ is the $\mathrm{H} \beta$ tomogram constructed from the DIS data (binned to $\Delta \phi=0.04)$; Figure $6 \mathrm{~h}$ is the $\mathrm{H} \beta$ tomogram for the combined set of RPCS spectra. The tomogram for 1995 Sep 19-20 UT (Fig. 6d) is of poorer quality than the others because the binned data set used to construct it (\#4 - see Table 1) contains substantially fewer total spectra than the other

RPCS data sets. As might be expected following the similar behavior of the average spectra (see $\S 3.1$ and emission line equivalent widths ( $(3.3)$, the $\mathrm{H} \beta$ tomogram does not change significantly from 1995 August to 1995 October. This implies that the changes in the radial velocity parameters of the emission lines must be due to small changes in the structure of a discrete emitting region rather than the whole disk.

There are two main features in the tomograms: (1) a roughly circular region of diffuse emission, centered around the velocity origin and extending to a radius of $\approx 600-800 \mathrm{~km} \mathrm{~s}^{-1}$; and (2) a localized region of strong emission centered at $V_{x} \approx-300$ to $-400 \mathrm{~km} \mathrm{~s}^{-1}$ and $V_{y} \approx 0$ to $+50 \mathrm{~km} \mathrm{~s}^{-1}$. The latter region is suggestive of the bright spot at the impact site of the accretion stream with the edge of the disk, but is somewhat inconsistent with the expected velocity position. Such emission would normally lie further inside the $\left(-V_{x},+V_{y}\right)$ quadrant of the tomogram (see, for example, Fig. 11 of Kaitchuck et al. 1994). In Figures 6g and 6h, we have plotted the positions of the secondary star Roche lobe and the accretion stream trajectory, using the system parameters 
determined by BSC94 (see \$1]). The strong emission region does not coincide with the stream trajectory as would be expected for normal bright spot emission. By arbitrarily reducing the WD orbital velocity from the value of $\approx 84 \mathrm{~km} \mathrm{~s}^{-1}$ calculated by BSC94 to $\approx 40 \mathrm{~km} \mathrm{~s}^{-1}$, we can force the accretion stream to pass roughly through the center of the emission region in the tomograms. However, this requires the masses of the stellar components to be unreasonably small $\left(M_{W D} \approx 0.07 M_{\odot}, M_{2} \approx 0.02 M_{\odot}\right)$ and, consequently, does not provide a useful explanation for the discrepancy between stream and emission locations.

The strong emission region also appears to be elongated towards the $\left(+V_{x},+V_{y}\right)$ quadrant; several of the tomograms (notably a, b, g, and h) show a "tail" of emission which extends from this region to the $+V_{y}$ axis, intersecting at $\approx+200 \mathrm{~km} \mathrm{~s}^{-1}$. This velocity is comparable to an estimate of the orbital velocity of the $L_{1}$ point around the center-of-mass assuming the mass ratio calculated by BSC94. Indeed, the velocity at the end of the emission tail on the $+V_{y}$-axis coincides with that of the tip of the secondary star Roche lobe (i.e., the $L_{1}$ point) as plotted in Figures $6 \mathrm{~g}$ and $6 \mathrm{~h}$; the tail itself follows the expected trajectory of the accretion stream.

None of the $\mathrm{H} \beta$ tomograms contains a distinct ring of emission (the signature of an accretion disk; e.g., see Fig. 24 of Kaitchuck et al. 1994), although there is some indication of such a structure in Figures 6c and 6f. Figure $6 \mathrm{~g}$ contains the most obvious hint of an emission ring. The lack of a prominent disk signature in the tomograms is not surprising given the single- rather than double-peaked emission lines in the spectrum of UU Aqr; this may be consistent with only a small disk being present, or only weak emission from a larger disk, or emission from a non-disk source (e.g., a wind) that masks the disk emission.

The $\mathrm{H} \gamma$ and, for the DIS spectra, $\mathrm{H} \alpha$ tomograms are very similar to the $\mathrm{H} \beta$ tomograms (the wavelength coverage of the RPCS spectra does not extend to $\mathrm{H} \alpha$ ). Representative examples of the tomograms of these lines, constructed from the DIS data, are shown in Figure $7 \mathrm{a}-\mathrm{b}$. The trailed spectrum used to construct the $\mathrm{H} \gamma$ tomogram is shown in Figure 8 (the trailed spectra for $\mathrm{H} \alpha$ and $\mathrm{H} \beta$ are shown in Fig. 9). There is a somewhat more distinct disk-ring of emission in the $\mathrm{H} \gamma$ tomogram than in the other Balmer tomograms; the disk may be more optically thin to the more energetic Balmer radiation (i.e., at hotter temperatures). The $\mathrm{H} \gamma$ ring emission is strongest along an arc starting at the strong emission region in the $\left(-V_{x},+V_{y}\right)$ quadrant and trailing counter-clockwise in the tomogram into the $\left(+V_{x},-V_{y}\right)$ quadrant, which suggests that the disk edge is nonuniform (in temperature, or density, or local turbelent velocity, etc.).

The spectra binned at a given phase from the DIS data set have somewhat higher signalto-noise than the corresponding binned RPCS spectra. Thus, we also attempted to construct Doppler tomograms for the weaker He I $\lambda 4471$ and He II $\lambda 4686$ lines in the DIS spectra. These are shown in Figure $0 \mathrm{dc}-\mathrm{d}$, and the trailed spectra for these lines are shown in Figure 8. The high velocity regions of the He I tomogram are severely contaminated by noise in the continuum adjacent to the line. However, there are still two features of note in the tomogram: (1) a region of enhanced emission on the $-V_{x}$ axis, consistent with the location of the one seen in the Balmer 
tomograms; and (2) a centralized lack of emission and slightly enhanced arc of emission spanning the $-V_{y}$ quadrants that are suggestive of the ring signature of disk emission. The He II tomogram is practically indistinguishable from noise, although there may be a slight enhancement of the emission level in the $\left(-V_{x},+V_{y}\right)$ quadrant (corresponding to the location of the strong emission region in the other tomograms). The poor quality of the He II tomogram is probably the result of both the weakness of the He II line and its severe blending with the adjacent C III/N III complex.

The only other published tomogram for UU Aqr is that of the $\mathrm{H} \beta$ line shown in the CV tomography atlas of Kaitchuck et al. (1994), and it is significantly different from our $\mathrm{H} \beta$ tomograms. The strong emission region in the Kaitchuck et al. tomogram is displaced upward to $V_{y} \approx+100$ to $+300 \mathrm{~km} \mathrm{~s}^{-1}$ and there is a much more prominent ring of disk emission (centered at $V_{y} \approx-200 \mathrm{~km} \mathrm{~s}^{-1}$ ) than in our tomograms. Overall, the Kaitchuck et al. tomogram of UU Aqr is much more similar to that of a dwarf nova type CV with a prominent, optically thin disk and a discrete bright spot at the expected site of the accretion stream impact with the outer edge of the disk. The Kaitchuck et al. tomogram was constructed using data obtained in 1988 October, during one of the photometric low states classified by BSC94. Thus, we may attribute the differences in the tomograms to differences in the accretion state of UU Aqr when the spectra were obtained: low for Kaitchuck et al., high for our data. The eclipse mapping of BSC94 shows that the disk in UU Aqr has a larger radius in the high state than in the low; however, the increased mass transfer in the high state may produce a strong wind emission component that masks the disk emission. The consistency of our $\mathrm{H} \beta$ tomogram from 1995 August to 1995 October implies that the physical conditions in the UU Aqr disk were very stable during the $\approx 3$ month span of our observations.

\section{Discussion}

\subsection{Line Profile Simulation}

As a simple model, CV emission line profiles can be simulated as the sum of several component profiles with the appropriate radial velocity vs. orbital phase behavior expected for various emission regions in the system (e.g., accretion stream, bright spot, WD, etc.). In this manner, Hellier \& Robinson (1994) and Hellier (1996) have had some success at reproducing the general line profile behavior of SW Sex stars (specifically, PX Andromedae and V1315 Aquilae). We have applied a similar technique to simulating the Balmer emission lines in UU Aqr. Figure 9 shows trailed spectrograms and Doppler tomograms for the observed $\mathrm{H} \alpha$ (DIS data) and $\mathrm{H} \beta$ (combined RPCS data) emission lines of UU Aqr, and a simulated Balmer line. The velocity and phase resolution of the simulated data was made comparable to that of the observed data. Our simulated line profiles are made up of five components, described here:

Double-peaked Keplerian disk emission: This is the only one of the components that is not represented by a Gaussian profile; instead, the disk profile is calculated as in Robinson, Marsh, \& Smak (1993), using an emissivity index $\beta=2.0$ (small changes in $\beta$ around this value 
do not have a significant effect on the simulation owing to the relatively small amplitude of the disk profile and the qualitative nature of the fitting process - see below). We used the system parameters and WD orbital velocity for UU Aqr derived by BSC94 to define the shape and orbital phase behavior of the disk profile. We initially assumed inner and outer disk radii of $0.015 \mathrm{R}_{\odot}$ and $0.4 \mathrm{R}_{\odot}$ (BSC94; Harrop-Allin \& Warner 1996). Subsequent comparison with the observed profiles (see Fig. 10) caused us to pick $0.045 \mathrm{R}_{\odot}$ and $0.45 \mathrm{R}_{\odot}$ as final inner and outer disk radii (the latter value is $\approx 60 \%$ of the distance from the WD to the $L_{1}$ point). These disk radii are used throughout the simulation. The amplitude of the disk profile in the simulation is 0.40 above the continuum level (which has been normalized to a value of 1.00 in the observed spectra).

Single-peaked WD/disk wind emission: This is represented by a Gaussian function with FWHM of $1000 \mathrm{~km} \mathrm{~s}^{-1}$ centered at the midpoint of the disk profile. It is assumed to follow the orbital motion of the WD. The amplitude of the wind component is 0.80 above the continuum. The amplitudes of both the disk and wind components were artificially enhanced by a factor of 2.25 during phases 0.98-0.05 to emulate the general appearance of the observed Balmer emission lines during eclipse. We have not explicitly calculated an eclipse profile for the simulated line (which would require determination of the precise 3-D locations of emission and continuum regions in the system). Hence, the treatment of the eclipse phases shown in the simulated trailed spectrogram in Figure 9 is, at best, only an approximation of the actual eclipse behavior. (We note, however, that even this simple approximation yields line profiles that are similar to the observed profiles during eclipse - see Fig. 10 - although it is not clear whether or not this is merely coincidental.) Since the data from the eclipse phases are excluded from the creation of a Doppler tomogram, any incorrect treatment of the simulated eclipse behavior has no effect on the simulated tomogram.

Accretion stream emission: The free-fall velocities along the accretion stream trajectory from the $\mathrm{L}_{1}$ point were calculated as in Lubow \& Shu (1975). Gaussian profiles with FWHM of $25 \%$ of the local stream velocity were summed along a specified length of this trajectory to simulate emission from the material in the stream. In addition, the Keplerian rotational velocities of the disk material underlying the stream trajectory were calculated, to provide the option of simulating disk material excited to emission through interaction with a stream that overflows the disk from its initial impact site. In the case of UU Aqr, we have assumed that the stream emits only between the $\mathrm{L}_{1}$ point and its impact with the edge of the disk - this reproduces the "tail" seen in the Balmer tomograms (see $\$ 3.4$ ). The peak amplitude of the stream component is 0.25 above the continuum.

Bright spot emission: In the model proposed by Hellier \& Robinson (1994) and Hellier (1996) for the SW Sex stars PX And and V1315 Aql, the accretion stream continues coherently past its initial impact with the edge of the disk, absorbing the underlying disk emission along its trajectory, to a secondary impact site in the inner disk (thereby creating a secondary bright spot whose velocity behavior is determined by the dynamics of the inner disk). The eclipse maps of UU Aqr in a high state (BSH96), however, show evidence for a bright spot only at the expected phase of the initial stream impact with the disk $(\phi \sim 0.8)$. Further, we find that the velocity of 
a secondary impact site near the radius expected for re-impact (Lubow 1989) must be arbitrarily reduced by a very large amount $(>50 \%)$ to match the observed velocity offset of the strong emission region in the Balmer tomograms of UU Aqr. Consequently, we have simulated the line profiles using a bright spot (Gaussian FWHM $=50 \%$ of the average of the local stream+disk rotation velocity) that originates at the intersection of the stream with the disk edge, and expands to lower velocities (this is consistent with, for example, emission from impacting stream material that does not deeply penetrate the disk and is primarily reflected away from the disk edge in the general direction of the secondary star). This scenario is suggested by recent numerical simulations of the stream-disk interaction (Armitage \& Livio 1996, 1997), in which the impact of the stream with the disk, in the presence of inefficient cooling (appropriate for high $\dot{M}$ systems such as novalike CVs), does not produce coherent stream flow over the disk, but instead causes an "explosion" of material away from the impact point in all directions (except those blocked by the disk). Thus, the stream component in the line simulation does not contribute beyond the outer edge of the disk. The peak amplitude of the bright spot profile is 1.20 above the continuum.

Non-axisymmetric absorption: Hellier (1997) has recently proposed accretion stream overflow onto an axisymmetrically flared disk to explain the transient absorption features in the emission lines of the SW Sex stars. Unfortunately, this mechanism appears only to be able to reproduce the absorption seen around $\phi \approx 0.5$ in some of the SW Sex stars. In order to account for absorption at both 0.5 and other phases, there must be a non-axisymmetric absorbing structure located at different positions in the disk in different systems, as suggested by Hoard \& Szkody (1996a, 1997). In order to match the observed behavior of the Balmer emission lines in UU Aqr, we utilized non-axisymmetric absorption represented by an inverted (i.e., negative amplitude) Gaussian function. The absorbing structure can be visualized as a vertically- and azimuthally-extended bulge or wall along the disk edge, which starts (with zero absorption) at $\phi=0.95$, ramps up linearly to peak absorption (amplitude of -0.63 relative to the continuum) between phases 0.7 and 0.8 , then ramps back down to zero absorption at $\phi=0.3$. The formation of such a structure could result from stream material "exploding" around the impact site (near $\phi \approx 0.8)$ to form the highest section of the wall, with additional material carried along the disk edge by the rotation of the disk to form the long, declining tail of the wall. This is consistent with the large azimuthal extent of the bright spot expected from the eclipse profiles of UU Aqr (see 3.3). The FWHM of the absorption is $300 \mathrm{~km} \mathrm{~s}^{-1}$; this value was initially set at the rotational velocity of the outer edge of the disk $\left(\approx 550 \mathrm{~km} \mathrm{~s}^{-1}\right)$ and was refined by comparing the simulated absorption width with that seen between phases 0.5 and 1.0 in the observed spectra. In the simulation shown in Figure 9, the velocity behavior of the absorption was set to follow that of the bright spot, in essence producing a self-absorption component of the bright spot (as suggested by Dickinson et al. 1997 to explain the line profile behavior of the probable SW Sex star V795 Herculis). We also experimented with somewhat more physical forms of velocity behavior for the absorption, such as following either the rotational velocity of the edge of the disk or the continuation of the stream trajectory over the disk, and found these to produce qualitatively similar results. Considering the simplicity of our simulation, we elected to present here the case 
involving the fewest unknown parameters (i.e., self-absorption).

In general, this technique of line profile simulation can produce reasonable-looking results. Yet, the large number of input parameters and use of simplifying approximations beg the question: how realistic are these results? We have calculated the $\chi^{2}$ values of the simulated profiles using different input parameters compared to the observed profiles, in a number of phase bins; the comparison for the simulation presented here is shown in Figure 10. This provided a means of comparing the relative "goodness" of simulations for a particular data set. However, the parameters were adjusted based only on a visual inspection of the simulated vs. real profiles; for example, the blue shoulder visible in the $\phi=0.15$ profile in Figure 10 provided a measure of the correct amplitudes for the disk and wind components, while the height of the central peak provided an estimate of the correct amplitude for the bright spot emission. This manual fitting can produce somewhat erratic results. Further, like Doppler tomography, the line profile simulation process essentially collapses the velocity field of the emission into two dimensions, which can lead to some ambiguity if significant sources of emission have large velocity components in the third dimension (i.e., vertical relative to the plane of the disk).

\subsection{Half-Orbit Tomography}

The presence of a non-axisymmetric absorbing structure in the disks of SW Sex stars appears to be quite successful at explaining the transient absorption features in the emission lines of these CVs. Unfortunately, it poses a potential problem for the technique of Doppler tomography, which is widely used in the analysis of both the SW Sex stars and CVs in general. A basic tenet of the tomography process is that the emission region(s) being mapped should be equally visible at all orbital phases of the system; this is why eclipse phase data are neither used in the tomography process nor accurately reproduced in forward-projections of the velocity maps. In order to circumvent the violation of this tenet in UU Aqr, we have constructed two additional sets of Balmer line tomograms. In the first set (shown in the top panels of Fig. 11), we used only the spectra from orbital phases 0.0 to 0.5 - the stronger, "unabsorbed" spectra (see top panels of Fig. 9). The second set uses the remaining, "absorbed" spectra, from $\phi=0.5-1.0$ (bottom panels of Fig. 11). Thus, each of these half-orbit time-resolved tomograms should show the emission from only one side of the absorbing structure described in $\$ 4.1$.

In the $\phi=0.0-0.5$ tomograms, we are effectively looking across most of the width of the disk to the absorbing structure on the far edge. The strong emission region lies on the $-V_{x}$ axis in

the same location as in the full-orbit tomograms. This emission originates at the explosive stream impact with the disk edge, and is viewed on the inner disk side of the absorbing structure. Little or no emission is visible along the accretion stream trajectory itself; the stream is likely obscured at most of these phases by the absorbing bulge, accretion disk, and/or secondary star. In the $\phi=0.5-1.0$ tomograms, we are looking only at the stream and the small amount of disk material at radii outside the absorbing structure. There is prominent emission along the accretion stream 
trajectory; however, there is little or no emission on the $-V_{x}$ axis. Presumably, most of the stream impact emission is obscured by the absorbing structure at these phases.

It was noted in $\$ 3.4$ that the $\mathrm{H} \gamma$ tomogram showed the most prominent suggestion of the ring indicative of an accretion disk. The half-orbit tomograms of this line show that the strongest part of this ring emission is visible only at $\phi=0.5-1.0$, in the form of enhanced emission that trails out along the disk-ring from the bright spot at the stream impact site. This corresponds to material carried along the outer edge of the disk by the disk rotation (e.g., Armitage \& Livio 1997); it is hidden behind the absorbing structure and/or secondary star for $\phi \approx 0.0-0.5$, so is not seen in the $\phi=0.0-0.5$ tomograms. A similar trail of emission is seen in tomograms of the archetype SW Sex (Dhillon, Marsh, \& Jones 1997) and the non-SW Sex system WZ Sagittae (Spruit \& Rutten 1997); Spruit \& Rutten suggest that this emission is due to recombination in material that is cooling down from the shock of the stream-disk impact as it is swept "downstream" by the disk rotation.

\subsection{A System Model}

Figure 12 is a schematic diagram of a CV showing the features of the model for UU Aqr suggested by our line profile simulations and half-orbit tomography. The high $\dot{M}$ accretion stream strikes the edge of the disk and forms a roughly spherical "explosion" of emitting material (region A in Fig. 12). This emitting material is flung primarily in directions of least resistance, away (and above) the disk, but some also disperses across the inner disk, producing diffuse emission in the tomograms (region B). An optically thick absorbing wall is built up by large amounts of the impacting stream material (region D), either carried along the disk edge or roughly following the continuation of the stream trajectory (although there is no coherent overflow of the stream).

Optically thin emitting gas heated by the shock of the stream impact is also carried along the very outer edge of the disk by the disk rotation, outside the absorbing structure (relative to the WD), forming the trailing arc of emission seen most prominently in the $\mathrm{H} \gamma$ tomograms (region $\mathrm{C}$ ). (This situation is likely to be further complicated by the probable presence of a wind from the disk that is mentioned, but not explicitly addressed, in this work.)

\subsection{UU Aqr as an SW Sex Star}

The definition of the SW Sex stars as a distinct class of CV is somewhat ambiguous and considerably more phenomenological than that of other CV classes (e.g., U Geminorum stars, polars, etc.) The SW Sex stars tend to be high inclination systems, but this is almost certainly a selection effect. They have similar orbital periods, $P_{\text {orb }} \approx 3-4 \mathrm{~h}$, which suggests a possible evolutionary link to the onset of SW Sex behavior. In general, they display single-peaked emission lines, orbital-phase-dependent absorption in the Balmer lines, and phase offsets in the emission line radial velocity curves. UU Aqr has all of these general qualities. Early work on the SW Sex 
stars (e.g., Szkody \& Piché 1990; Thorstensen et al. 1991) specified that the absorption occurred only at $\phi \approx 0.5$, whereas it occurs at $\phi \approx 0.8$ in UU Aqr (and in PG 0859+415). Yet, this variation in behavior can be accommodated if the SW Sex "class" contains systems comprising a continuum of observational characteristics determined by the relative values of fundamental parameters (such as the mass transfer rate), rather than many examples of a fixed system morphology.

We note that strong He II $\lambda 4686$ emission (relative to the Balmer emission lines, for example) is also typically mentioned as a distinguishing property of the SW Sex stars, but UU Aqr has weak He II. The strength of He II, however, does vary among the members of this class (Hoard \& Szkody 1996b), from comparable to $\mathrm{H} \beta$ (e.g., DW UMa; Shafter et al. 1988) to much weaker than $\mathrm{H} \beta$ (e.g., WX Arietis; Beuermann et al. 1992). Thus, although the reason for the weak He II emission in UU Aqr is not entirely clear, just the fact that it is weak is not so troubling. It is reasonable to suggest that the strength of He II is related to inclination; for example, WX Ari ( $i \lesssim 75^{\circ}$; Beuermann et al. 1992) and PG 0859+415 ( $i \approx 65^{\circ}$; Hoard \& Szkody 1996a), which are both moderate inclination systems, have weak He II emission. However, this correlation does not appear to extend to other systems: BH Lyn $\left(i=79^{\circ}\right.$; Hoard \& Szkody 1997) and SW Sex $\left(i=79^{\circ}\right.$; Penning et al. 1984) have inclinations similar to that of UU Aqr but have strong He II emission, while PX And $\left(i=73.6^{\circ}\right.$; Thorstensen et al. 1991) has a smaller inclination than UU Aqr, but stronger He II emission. The presence of optically thick parts of the outer disk that obscure the hot inner regions where He II is expected to form might reduce the emission strength; for example, PG 0859+415 and UU Aqr are known or suspected of having thick disks and both display weak He II emission.

The dependency of the amount of stream overflow on cooling efficiency noted by Armitage \& Livio (1997) provides at least a partial explanation for the range of behavior among the SW Sex stars. At high mass transfer rates, cooling is inefficient and the stream impact produces a forceful explosion, resulting in no coherent stream overflow and the presence of line absorption at the initial impact site $(\phi \approx 0.8)$. At low mass transfer rates, cooling is efficient and there is substantial, coherent overflow of the stream. This allows the formation of a bright spot in the inner disk at the site of the secondary stream impact site, resulting in line absorption around $\phi \approx 0.5$. In the SW Sex stars UU Aqr and PG0859+415 (Hoard \& Szkody 1996a), the line absorption is deepest near $\phi=0.8$ and there is evidence for a substantial bright spot on the edge of the disk. In BH Lyn, the absorption occurs around $\phi=0.5$ but there is also evidence for the presence of a bright spot on the edge of the disk from time to time (Hoard \& Szkody 1997). In DW UMa (Shafter et al. 1988), PX And (Thorstensen et al. 1991), and V1315 Aql (Dhillon, Marsh, \& Jones 1991), the absorption occurs around $\phi=0.5$ and there is little or no evidence for a bright spot on the disk edge. We can understand this range of behavior in the context of the numerical simulations of Armitage \& Livio (1997) if the mass transfer rate is largest for UU Aqr and PG $0859+415, \dot{M}$ is smaller for $\mathrm{BH}$ Lyn, and still smaller for DW UMa, PX And, and V1315 Aql. This produces primarily explosive impact of the stream with the disk in the first two systems, a mix of explosive impact and stream overflow in BH Lyn, and primarily stream overflow in the last three systems. Mass transfer rates 
in CVs are notoriously difficult to estimate, but we note that if their $\dot{M}$ 's do compare as described here, then the orbital periods of these six systems (UU Aqr $=3.9 \mathrm{~h} ; \mathrm{PG} 0859+415=3.7 \mathrm{~h}$; BH Lyn $=3.7 \mathrm{~h} ;$ PX And $=3.5 \mathrm{~h} ; \mathrm{V} 1315 \mathrm{Aql}=3.3 \mathrm{~h} ; \mathrm{DW} \mathrm{UMa}=3.3 \mathrm{~h}$ ) roughly follow a trend where mass transfer rate decreases with decreasing orbital period. Some evidence for such a trend has been seen among the novalike CVs (Dhillon 1996).

We wish to thank Keith Horne for making his Doppler tomography software available to us, and Stefanie Wachter for reading a draft of this paper. The research of PS and DWH was supported by NASA grant NAG-W-3158 and NSF grant AST9217911. MDS was supported by the Particle Physics and Astronomy Research Council grant K46019. The work of RCS was partially supported by PPARC grant GR/K45555.

\section{REFERENCES}

Armitage, P. J. \& Livio, M. 1996, ApJ, 470, 1024

Armitage, P. J. \& Livio, M. 1997, preprint

Baptista, R., Steiner, J. E., \& Cieslinski, D. 1994, ApJ, 433, 332 (= BSC94)

Baptista, R., Steiner, J. E., \& Horne, K. 1996, MNRAS, 282, 99 (= BSH96)

Beljawsky, P. R. 1926, Beobachtungs-Zircular der Astr. Nach., 6, 38

Beuermann, K., Thorstensen, J. R., Schwope, A. D., Ringwald, F. A., \& Sahin, H. 1992, A\&A, 256,442

Cousins, A. W. J. 1987, South African Astron. Obs. Circ., 11, 93

Dhillon, V. S. 1996, in Cataclysmic Variables and Related Objects, eds. A. Evans and J. H. Wood (The Netherlands: Kluwer), 3

Dhillon, V. S., Jones, D. H. P., \& Marsh, T. R. 1994, MNRAS, 266, 859

Dhillon, V. S., Marsh, T. R., \& Jones, D. H. P. 1997, MNRAS, in press

Dhillon, V. S., Marsh, T. R., \& Jones, D. H. P. 1991, MNRAS, 252, 342

Diaz, M. P. \& Steiner, J. E. 1991, AJ, 102, 1417 (= DS91)

Dickinson, R. J., Prinja, R. K., Rosen, S. R., King, A. R., Hellier, C. \& Horne, K. 1997, MNRAS, 286,447

Garnavich, P. M., Szkody, P., Mateo, M., Feinswog, L., Booth, J., Goodrich, B., Miller, H. R., Carini, M. T., \& Wilson, J. W. 1990, ApJ, 365, 696

Goldader, J. D. \& Garnavich, P. 1989, IBVS, no. 3361

Haefner, R. 1989, IBVS, no. 3397 
Hamuy, M., Suntzeff, N. B., Heathcote, S. R., Walker, A. R., Gigoux, P., \& Phillips, M. M. 1994, PASP, 106, 566

Harrop-Allin, M. K. \& Warner, B. 1996, MNRAS, 279, 219

Hellier, C. 1996, ApJ, 471, 949

Hellier, C. 1997, preprint

Hellier, C. \& Robinson, E. L. 1994, ApJ, 431, L107

Hirose, M., Osaki, Y., \& Mineshige, S. 1991, PASJ, 43, 809

Hoard, D. W. \& Szkody, P. 1996a, ApJ, 470, 1052

Hoard, D. W. \& Szkody, P. 1996b, BAAS, 28, 1371

Hoard, D. W. \& Szkody, P. 1997, ApJ, 481, 433

Honeycutt, R. K., Adams, B. R., Swearingen, D. J., \& Kopp, W. R. 1994, PASP, 106, 670

Honeycutt, R. K. \& Turner, G. W. 1992, in Robotic Telescopes in the 1990's: ASP Conference Series, Vol. 34, ed. A. V. Filippenko (San Francisco: ASP), 77

Horne, K. 1991, in Fundamental Properties of Cataclysmic Variable Stars: Proceedings of the 12th North American Workshop on Cataclysmic Variables and Low Mass X-ray Binaries, ed. A. W. Shafter (San Diego: San Diego State University), 23

Kaitchuck, R. H., Schlegel, E. M., Honeycutt, R. K., Horne, K., Marsh, T. R., White, J. C. II, \& Mansperger, C. S. 1994, ApJS, 93, 519

King, A. R. 1988, QJRAS, 29, 1

Lubow, S. H. 1989, ApJ, 340, 1064

Lubow, S. H. \& Shu, F. H. 1975, ApJ, 198, 383

Marsh, T. R. \& Horne, K. 1988, MNRAS, 235, 269

Massey, P., Strobel, K., Barnes, J. V., \& Anderson, E. 1988, ApJ, 328, 315

Massey, P., Valdes, F., \& Barnes, J. 1992, A User's Guide to Reducing Slit Spectra with IRAF, available on-line from the NOAO at http://iraf.noao.edu/

Meglicki, Z., Wickramasinghe, D., \& Bicknell, G. V. 1993, MNRAS, 264, 691

Menzies, J. W. \& Glass, I. S. 1996, Ap\&SS, 235, 175

Oke, J. B. \& Wade, R. A. 1982, AJ, 87, 670

Payne-Gaposchkin, C. 1952, Ann. Astron. Obs. Harvard College, 115, no. 22

Penning, W. R., Ferguson, D. H., McGraw, J. T., \& Liebert, J. 1984, ApJ, 276, 233

Pringle, J. E. 1981, ARA\&A, 19, 137

Robertson, J. W. 1997, private communication 
Robinson, E. L., Marsh, T. R., \& Smak, J. 1993, in Accretion Disks in Compact Stellar Systems, J. C. Wheeler ed. (River Edge, NJ: World Scientific Publishing), 75

Rutten, R. G. M., van Paradijs, J., \& Tinbergen, J. 1992, A\&A, 260, 213

Schneider, D. P. \& Young, P. 1980, ApJ, 238, 946

Shafter, A. W., Hessman, F. V., \& Zhang, E. H. 1988, ApJ, 327, 248

Shafter, A. W., Szkody, P., \& Thorstensen, J. R. 1986, ApJ, 308, 765

Spruit, H. C. \& Rutten, R. G. M. 1997, A\&A, in press

Still, M. D., Dhillon, V. S., \& Jones, D. H. P. 1995, MNRAS, 273, 863

Szkody, P. 1985, in Recent Results on Cataclysmic Variables: Proceedings of the ESA Workshop, ed. W. R. Burke (Bamberg: ESA SP-236), 39

Szkody, P. \& Piché, F. 1990, ApJ, 361, 235

Thorstensen, J. R., Ringwald, F. A., Wade, R. A., Schmidt, G. D., \& Norsworthy, J. E. 1991, AJ, 102,272

Volkov, I. M., Shugarov, S. Y., \& Seregina, T. M. 1986, Astr. Tsirk., 1418, 3 


\section{FIGURE CAPTIONS}

Fig. 1.- Average red (bottom) and blue (top) spectrum of UU Aqr, uncorrected for orbital motion. The red spectrum is the DIS data from 1995 Oct 12-13 UT. The blue spectra are from (top to bottom) 1995 Aug 1-3 UT, 1995 Aug 6-8 UT, 1995 Aug 18-20 UT, 1995 Sep 19-20 UT, 1995 Sep 21-23 UT, 1995 Sep 24-26 UT, 1995 Oct 12-13 UT. The blue flux scale refers to the bottom spectrum (DIS data); the other spectra have been cumulatively offset by $0.5 \times 10^{-14} \mathrm{erg} \mathrm{s}^{-1} \mathrm{~cm}^{-2}$ $\AA^{-1}$.

Fig. 2.- Diagnostic diagrams from the double Gaussian fitting technique for the DIS data. The left panels are for the Balmer lines: $\mathrm{H} \alpha$ (dotted line), $\mathrm{H} \beta$ (solid line), and $\mathrm{H} \gamma$ (dashed line). The right panels are for the helium lines: He I $\lambda 4471$ (dotted line), He II $\lambda 4686$ (solid line), and He I $\lambda 5876$ (dashed line). The panels show (from bottom to top) the phase shift of the velocity solution, the systemic velocity $\left(\mathrm{km} \mathrm{s}^{-1}\right)$, the fractional uncertainty of the velocity semi-amplitude, the velocity semi-amplitude $\left(\mathrm{km} \mathrm{s}^{-1}\right)$, and the total $1 \sigma$ uncertainty of the velocity fit $\left(\mathrm{km} \mathrm{s}^{-1}\right)$.

Fig. 3.- Emission line radial velocity curves measured from the wings of the DIS spectra using the double Gaussian fitting technique.

Fig. 4.- Equivalent width curves of the prominent emission lines in the DIS spectra of UU Aqr. The top right panel shows the same $\mathrm{H} \alpha$ data as the top left panel plotted on an expanded scale to show the variation of the equivalent width outside of eclipse.

Fig. 5.- Light curve of UU Aqr obtained on 1995 August 01-02 and 06-07 UT. The top panel shows the entire curve, phased according to the ephemeris of BSC94, while the bottom panel shows a close-up of the region around the eclipse, with data obtained during different orbital cycles plotted with differently-shaped points.

Fig. 6.- Doppler tomograms for the $\mathrm{H} \beta$ emission line of UU Aqr from 1995 August to 1995 October. Panels (a-f) were constructed using the binned RPCS data sets from (a) 1995 Aug 1-3 UT, (b) 1995 Aug 6-8 UT, (c) 1995 Aug 18-20 UT, (d) 1995 Sep 19-20 UT, (e) 1995 Sep 21-23 UT, and (f) 1995 Sep 24-26 UT. Panel (g) was constructed using the binned DIS data set. Panel (h) was constructed using the combined set of RPCS spectra. The secondary star Roche lobe and accretion stream trajectory are plotted in panels $(\mathrm{g})$ and $(\mathrm{h})$ using the system parameters determined by BSC94.

Fig. 7.- Doppler tomograms for several emission lines constructed from the DIS data: (a) H $\alpha$, (b) $\mathrm{H} \gamma$, (c) He I $\lambda 4471$, (d) He II $\lambda 4686$ (+ C III/N III).

Fig. 8. - Trailed spectra for the He II + C III/N III emission complex (left), the He I $\lambda 4471$ emission line (middle), and the $\mathrm{H} \gamma$ emission line (right). Emission is dark; continuum is light. The spectra are repeated over two orbital cycles. Note that the velocity scale of the He II panel is larger than that of the other two panels to show the adjacent $\mathrm{C}$ III/N III emission. 
Fig. 9.- Trailed spectrograms and Doppler tomograms of UU Aqr. The left panels show the $\mathrm{H} \alpha$ line from the DIS spectra. The right panels show the $\mathrm{H} \beta$ line from the total combined RPCS data set. The middle panels show simulated data. The Roche lobe of the secondary star, the path of the accretion stream (lower curve), and the position of Keplerian disk velocities under the stream trajectory (upper curve) have been plotted in the Doppler tomograms, based on the system parameters determined by BSC94.

Fig. 10.- Observed (solid line) and simulated (dotted line) orbital-phase-resolved emission line profiles for UU Aqr. The observed data is the $\mathrm{H} \alpha$ line from the DIS spectra.

Fig. 11.- Half-orbit tomograms for the $\mathrm{H} \alpha$ (left panels), $\mathrm{H} \beta$ (middle panels), and $\mathrm{H} \gamma$ (right panels) emission lines of UU Aqr. The DIS data were used for $\mathrm{H} \alpha$ and $\mathrm{H} \gamma$; the RPCS data for $\mathrm{H} \beta$. The tomograms in the top panels were constructed using only the spectra in the range $0.0 \leq \phi \leq 0.5$; those in the lower panels used only the spectra in the range $0.5 \leq \phi \leq 1.0$. The linear structures visible in the tomograms are artifacts caused by the decreased orbital phase coverage of the input spectra.

Fig. 12.- A schematic model of UU Aqr. The Roche-lobe-filling secondary star is shown on the right, and the white dwarf (black dot) and accretion disk are on the left. The orbital phases of a number of lines-of-sight through the system are labeled around the perimeter of the dashed circle enclosing the diagram. Phase 0.0 corresponds to the superior conjunction of the WD. The system rotates counter-clockwise in its orbit, which corresponds to an observer moving clockwise around the lines of sight down each fiducial phase. The lettered regions are described in $\S 4.3$ of the text. 
Table 1. Observation Log

\begin{tabular}{|c|c|c|c|c|c|c|c|}
\hline $\begin{array}{c}\text { Binned } \\
\text { Data Set }\end{array}$ & $\begin{array}{c}\text { mid-JD of } \\
\text { Binned Data }\end{array}$ & Instrument & UT Date & $\begin{array}{r}\mathrm{UT}^{\prime} \\
\text { Start }\end{array}$ & $\begin{array}{l}\text { Time } \\
\text { End }\end{array}$ & $\begin{array}{l}\text { Orbital } \\
\text { Phases }\end{array}$ & $\begin{array}{c}\text { \# of } \\
\text { Spectra }\end{array}$ \\
\hline \multirow[t]{2}{*}{1} & \multirow[t]{2}{*}{2449932} & RPCS & 1995 Aug 01-02 & $21: 24$ & 02:04 & $0.51-1.70$ & 161 \\
\hline & & RPCS & 1995 Aug 02-03 & $22: 16$ & $02: 28$ & $0.84-1.91$ & 146 \\
\hline \multirow[t]{2}{*}{2} & \multirow[t]{2}{*}{2449937} & RPCS & 1995 Aug 06-07 & $21: 33$ & $02: 59$ & $0.11-1.50$ & 187 \\
\hline & & RPCS & 1995 Aug 07-08 & $21: 58$ & 03:00 & $0.33-1.61$ & 172 \\
\hline \multirow[t]{2}{*}{3} & \multirow[t]{2}{*}{2449949} & RPCS & 1995 Aug 18-19 & $22: 44$ & $02: 40$ & $0.77-1.78$ & 145 \\
\hline & & RPCS & 1995 Aug $19-20$ & $22: 43$ & $02: 18$ & $0.88-1.80$ & 122 \\
\hline \multirow[t]{2}{*}{4} & \multirow[t]{2}{*}{2449980} & RPCS & 1995 Sep 19-20 & $23: 20$ & $01: 21$ & $0.55-1.06$ & 69 \\
\hline & & RPCS & 1995 Sep 20 & $20: 05$ & $22: 58$ & $0.83-1.57$ & 91 \\
\hline \multirow[t]{2}{*}{5} & \multirow[t]{2}{*}{2449982} & RPCS & 1995 Sep 21-22 & $20: 16$ & $00: 37$ & $0.99-2.10$ & 146 \\
\hline & & RPCS & 1995 Sep 22-23 & $23: 13$ & $00: 56$ & $0.86-1.29$ & 58 \\
\hline \multirow[t]{2}{*}{6} & \multirow[t]{2}{*}{2449984} & RPCS & 1995 Sep 24-25 & $21: 43$ & 01:05 & $0.70-1.56$ & 111 \\
\hline & & RPCS & 1995 Sep 25-26 & $22: 40$ & $00: 58$ & $0.06-1.64$ & 84 \\
\hline \multirow[t]{2}{*}{7} & \multirow[t]{2}{*}{2450003} & DIS & 1995 Oct 12 & $04: 45$ & $08: 18$ & $0.41-1.31$ & 35 \\
\hline & & DIS & 1995 Oct 13 & 05:06 & 08:07 & $0.61-1.38$ & 30 \\
\hline
\end{tabular}


Table 2. Radial Velocity Parameters from Emission Line Wings

\begin{tabular}{|c|c|c|c|c|c|c|c|c|c|c|}
\hline $\begin{array}{l}\text { Data } \\
\text { Set }\end{array}$ & $\begin{array}{l}\mathrm{H} \beta: \\
\gamma^{\mathrm{a}} \\
\left(\mathrm{km} \mathrm{s}^{-1}\right)\end{array}$ & $\begin{array}{c}K \\
\left(\mathrm{~km} \mathrm{~s}^{-1}\right)\end{array}$ & $\phi_{0}$ & $\sigma_{K} / K$ & $\begin{array}{c}\sigma_{\text {TOTAL }} \\
\left(\mathrm{km} \mathrm{s}^{-1}\right)\end{array}$ & $\begin{array}{l}\mathrm{H} \gamma: \\
\quad \gamma^{\mathrm{a}} \\
\left(\mathrm{km} \mathrm{s}^{-1}\right)\end{array}$ & $\begin{array}{c}K \\
\left(\mathrm{~km} \mathrm{~s}^{-1}\right)\end{array}$ & $\phi_{0}$ & $\sigma_{K} / K$ & $\begin{array}{c}\sigma_{\text {TOT AL }} \\
\left(\mathrm{km} \mathrm{s}^{-1}\right)\end{array}$ \\
\hline 1 & $36(2)$ & $117(12)$ & $0.15(2)$ & 0.10 & 43 & $27(4)$ & $119(8)$ & $0.15(2)$ & 0.07 & 36 \\
\hline 2 & $8(1)$ & $113(18)$ & $0.14(2)$ & 0.16 & 59 & $15(3)$ & $107(15)$ & $0.11(2)$ & 0.14 & 48 \\
\hline 3 & $4(4)$ & $114(12)$ & $0.16(2)$ & 0.11 & 50 & $81(5)$ & $97(11)$ & $0.18(2)$ & 0.11 & 45 \\
\hline 4 & $86(5)$ & $95(11)$ & $0.13(3)$ & 0.12 & 45 & $65(10)$ & $117(22)$ & $0.12(4)$ & 0.19 & 78 \\
\hline 5 & $8(6)$ & $77(12)$ & $0.10(3)$ & 0.16 & 49 & $45(4)$ & $121(19)$ & $0.15(3)$ & 0.16 & 67 \\
\hline 6 & $31(4)$ & $113(16)$ & $0.09(2)$ & 0.14 & 51 & $31(8)$ & $104(12)$ & $0.08(3)$ & 0.11 & 51 \\
\hline 7 & $-19(1)$ & $115(6)$ & $0.13(1)$ & 0.05 & 34 & $18(2)$ & $103(8)$ & $0.10(1)$ & 0.08 & 43 \\
\hline 7 & $\begin{array}{l}\mathrm{H} \alpha: \\
\quad-4(1)\end{array}$ & $151(8)$ & $0.21(1)$ & 0.05 & 38 & \multicolumn{5}{|c|}{ He I $\lambda 5876:$} \\
\hline & \multicolumn{5}{|c|}{ He I $\lambda 4471:{ }^{\mathrm{c}}$} & \multicolumn{5}{|c|}{ He II $\lambda 4686:$ b,c } \\
\hline 7 & 33 & 156 & 0.13 & 0.16 & 135 & -87 & 226 & 0.27 & 0.15 & 165 \\
\hline
\end{tabular}

${ }^{\text {a} H e l i o c e n t r i c ~ c o r r e c t i o n s ~ a p p l i e d ~ t o ~ s y s t e m i c ~ v e l o c i t i e s . ~}$

${ }^{\mathrm{b}}$ Blended with C III/N III emission.

${ }^{\mathrm{c}}$ Weighted average of velocity parameter values in the range $a=1100-1800 \mathrm{~km} \mathrm{~s}^{-1}-$ see text. 
Table 3. Mean Emission Line Equivalent Widths $(0.1 \leq \phi \leq 0.9)$

\begin{tabular}{cccccc}
\hline \hline \multirow{2}{*}{$\begin{array}{c}\text { Data } \\
\text { Set }\end{array}$} & UT Date & $\mathrm{H} \beta$ & $\mathrm{H} \gamma$ & $\mathrm{He}$ I $\lambda 4471$ & $\mathrm{He}$ II $\lambda 4686^{\text {a }}$ \\
\hline \multirow{2}{*}{1} & 1995 Aug 01-03 & $18.2(1.1)$ & $12.1(2.0)$ & $1.4(0.7)$ & $9.8(1.6)$ \\
2 & 1995 Aug 06-08 & $18.3(2.3)$ & $12.7(1.4)$ & $2.1(0.8)$ & $9.7(1.4)$ \\
3 & 1995 Aug 18-20 & $21.1(3.4)$ & $11.8(2.5)$ & $2.1(1.4)$ & $6.7(1.8)$ \\
4 & 1995 Sep 19-20 & $19.0(3.0)$ & $13.3(7.5)$ & $2.0(1.9)$ & $8.2(5.7)$ \\
5 & 1995 Sep 21-23 & $21.9(3.6)$ & $13.9(2.2)$ & $2.6(1.2)$ & $4.6(5.5)$ \\
6 & 1995 Sep 24-26 & $17.6(2.1)$ & $12.1(3.2)$ & $2.7(1.2)$ & $8.5(1.8)$ \\
7 & 1995 Oct 12-13 & $17.2(2.8)$ & $9.2(2.5)$ & $0.0(1.0)$ & $10.2(1.9)$ \\
\hline
\end{tabular}

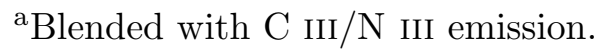



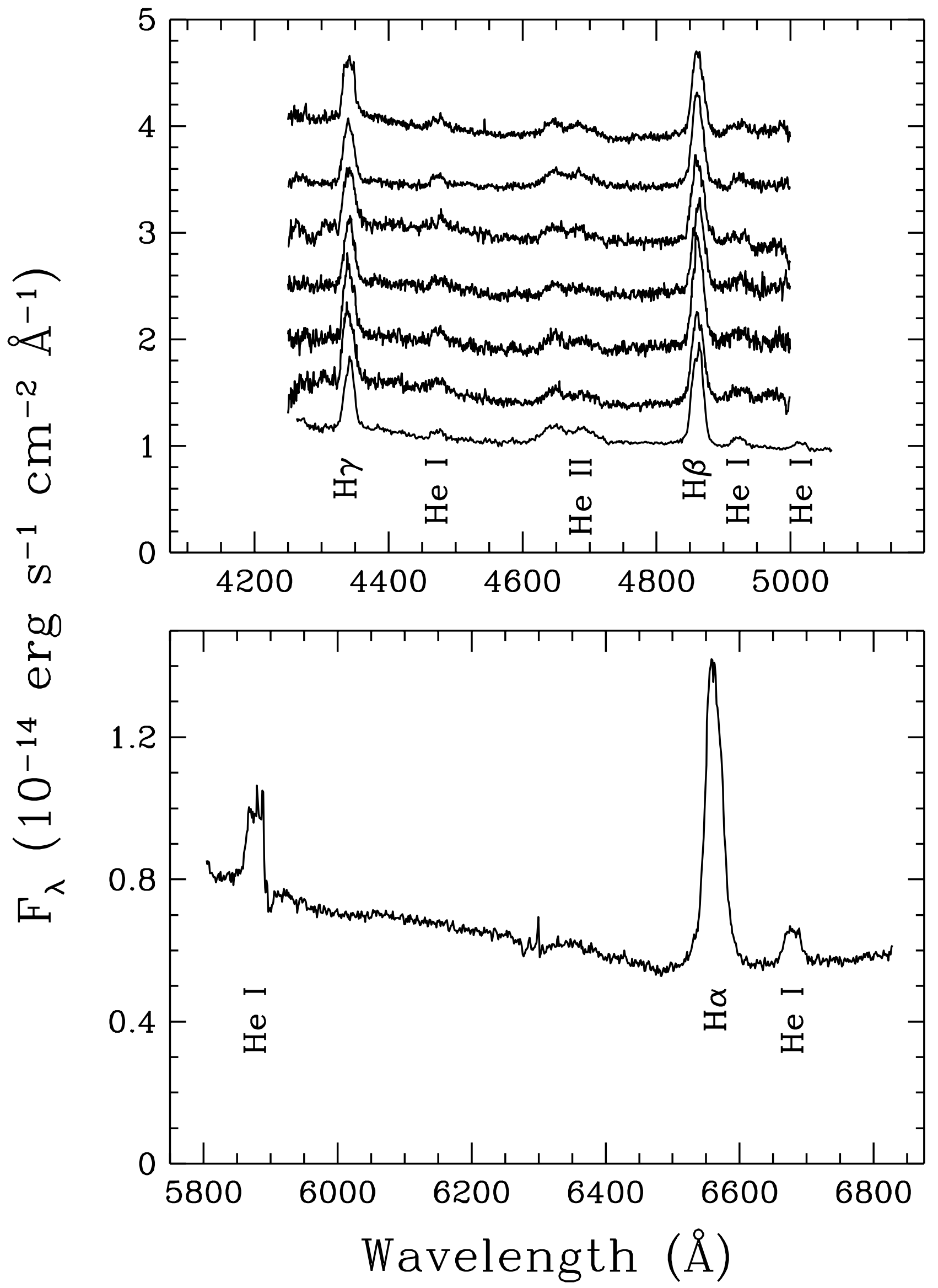


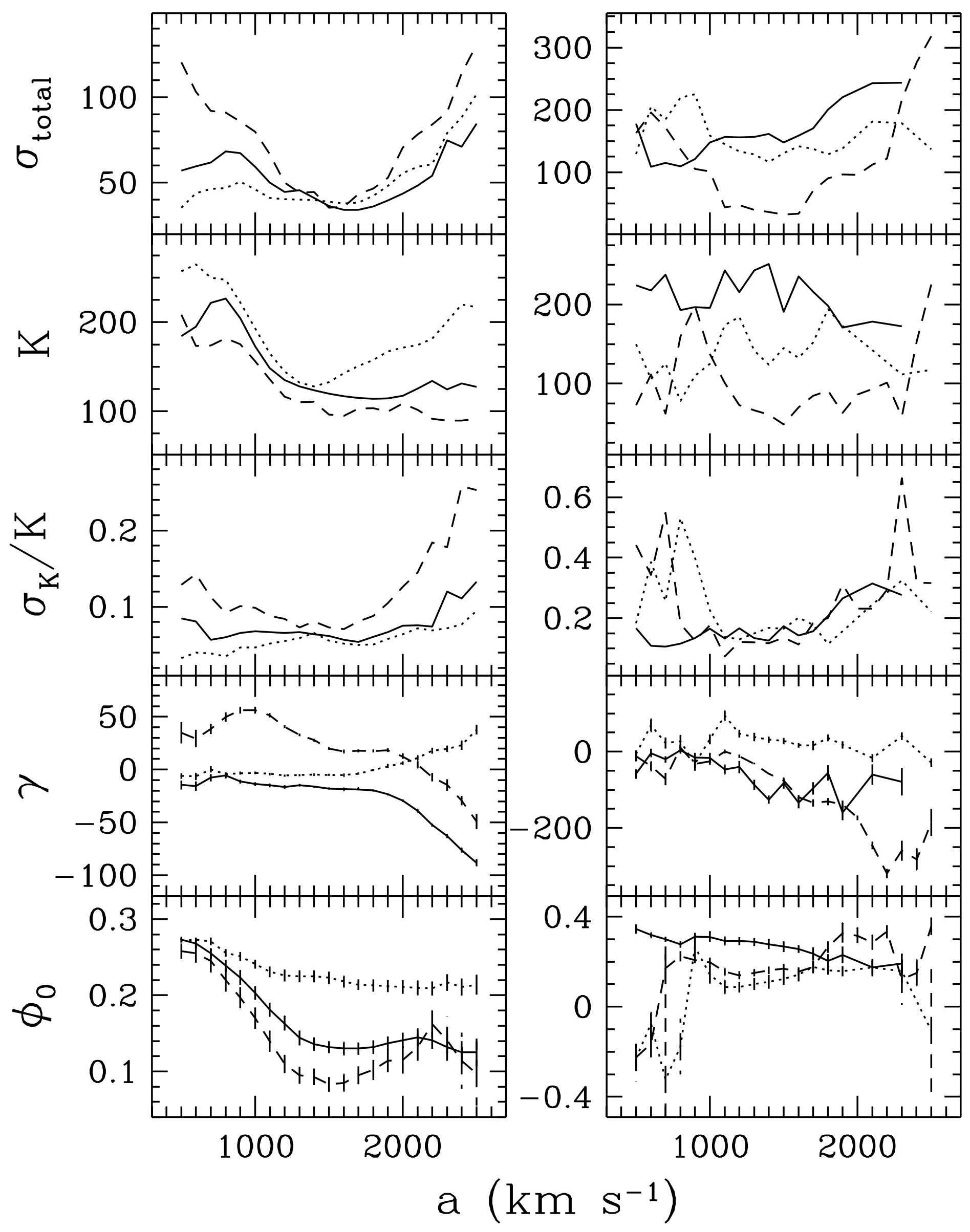




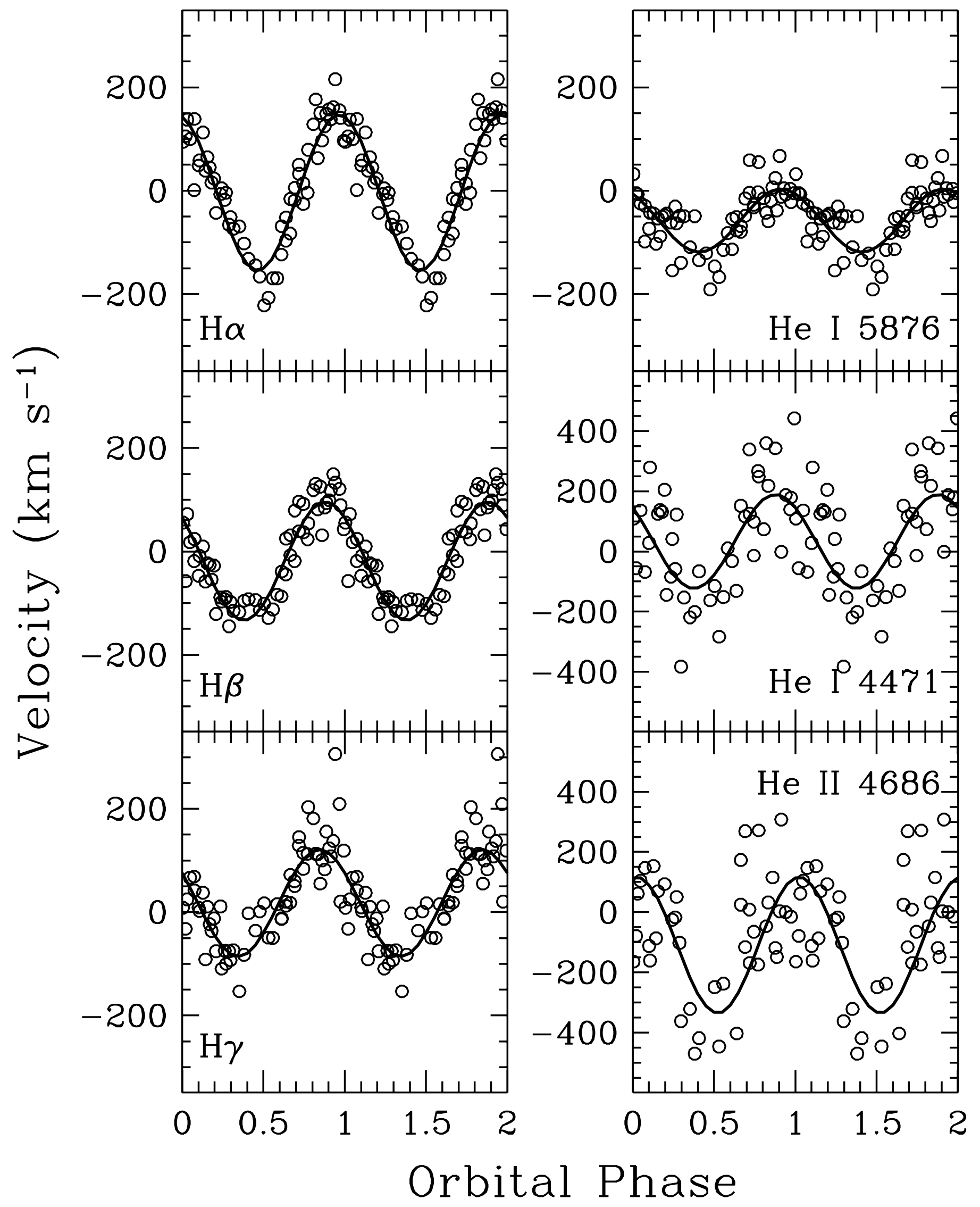




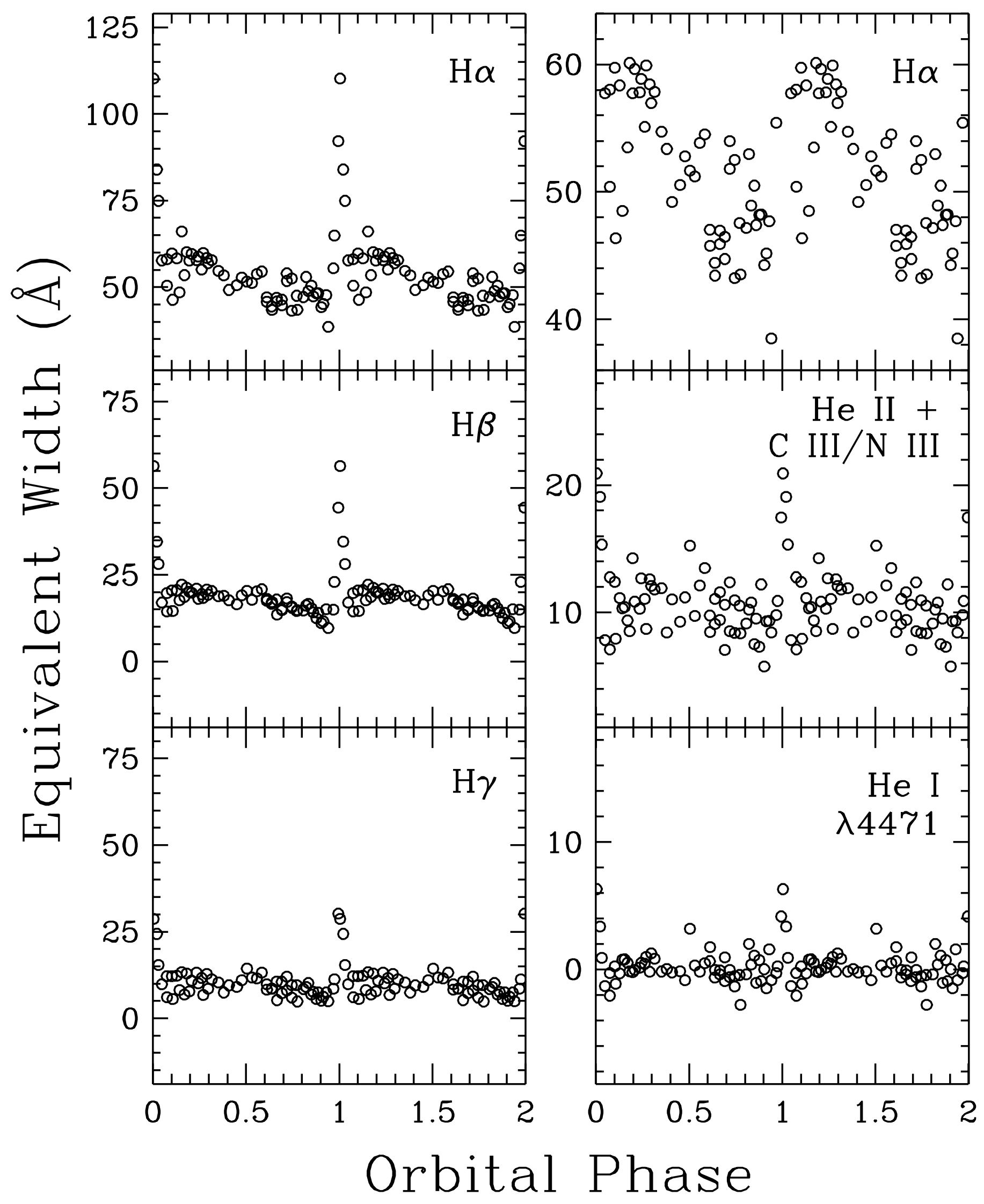




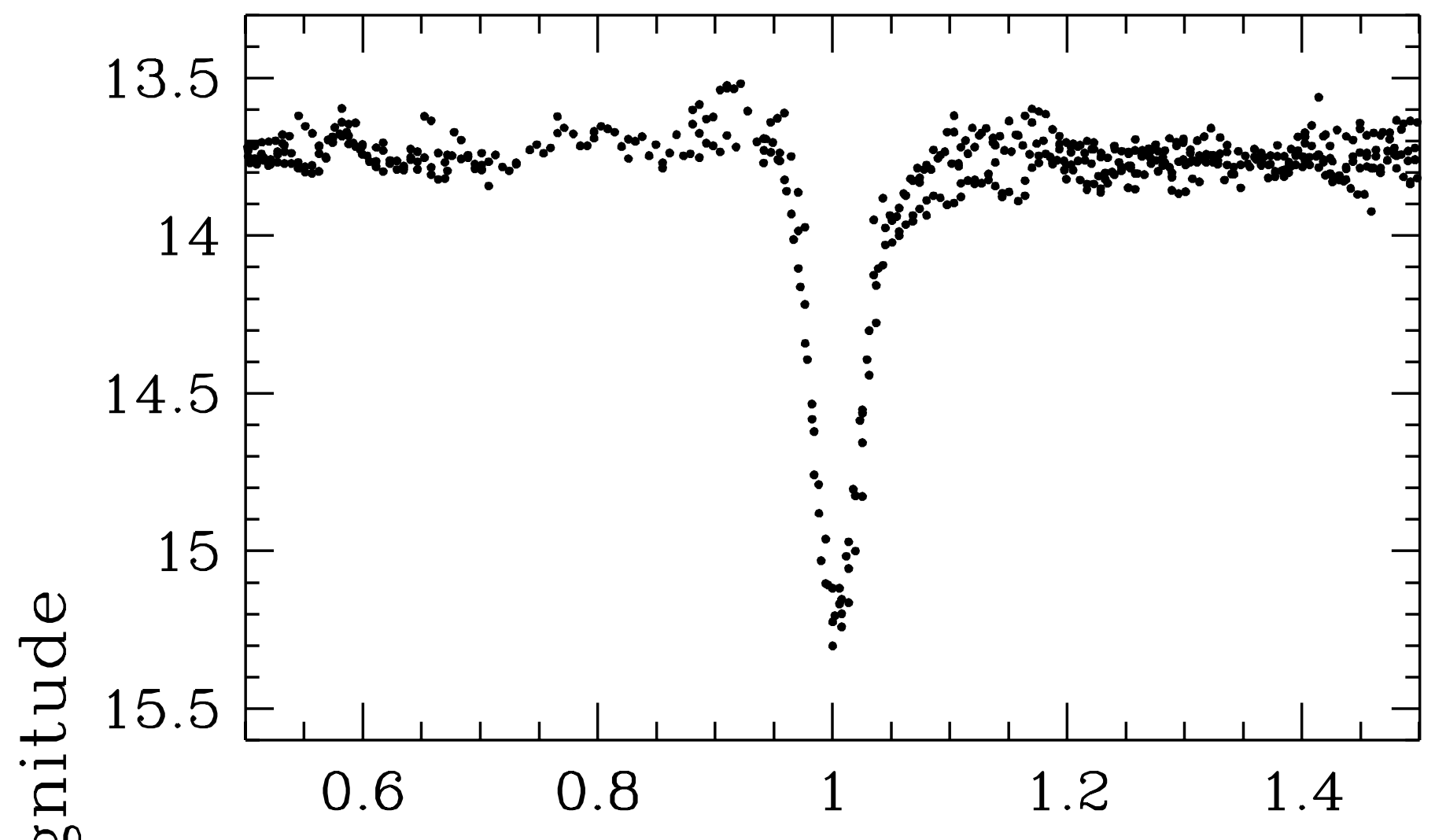

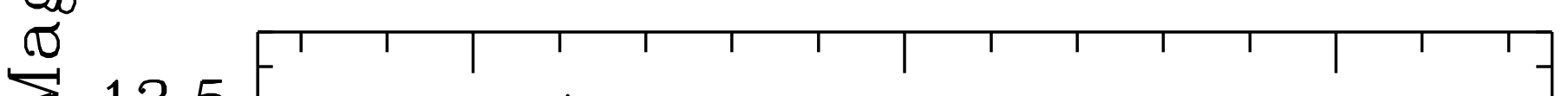

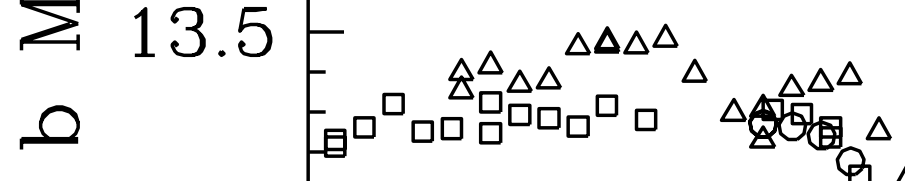

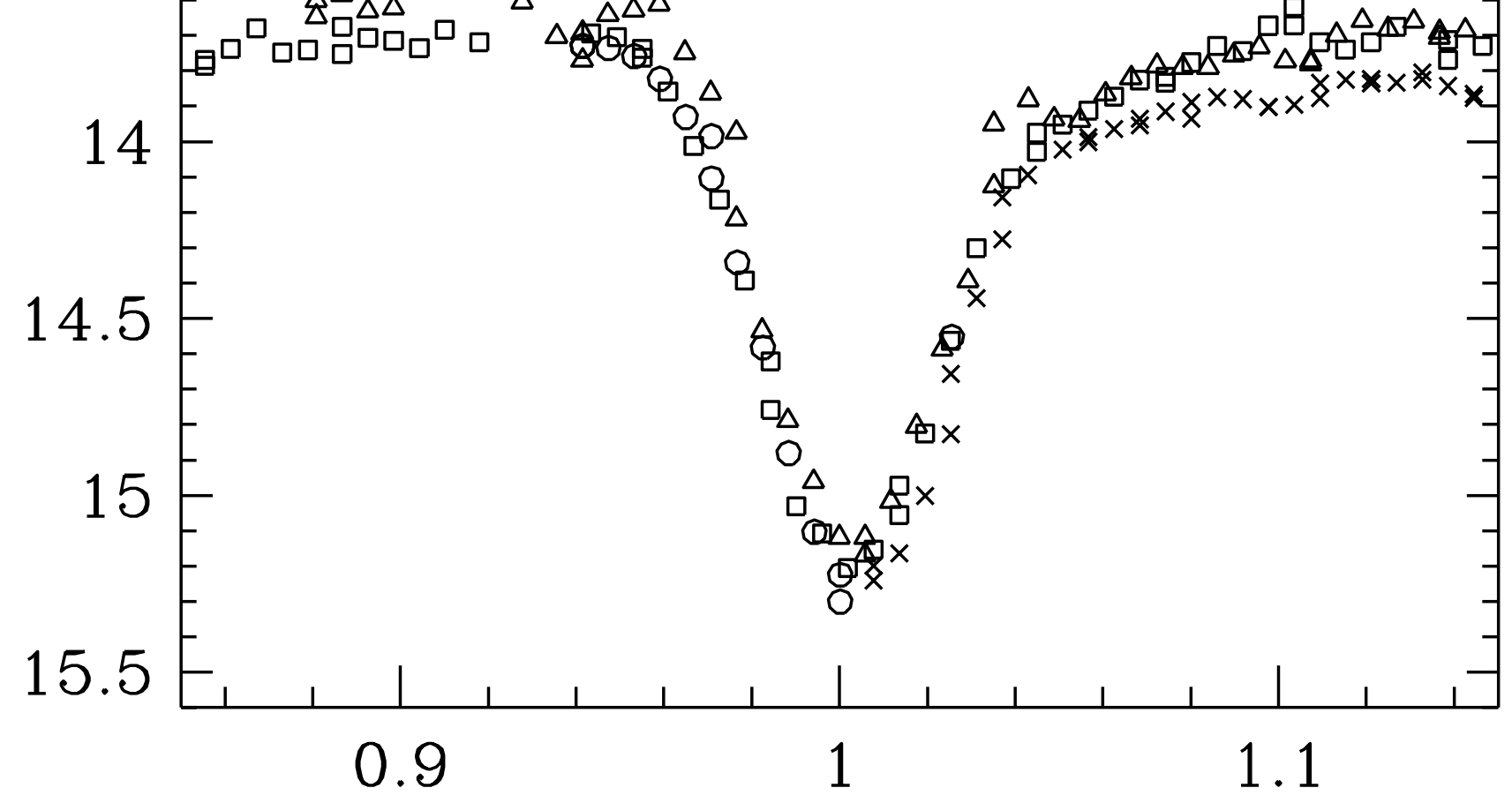

Orbital Phase 


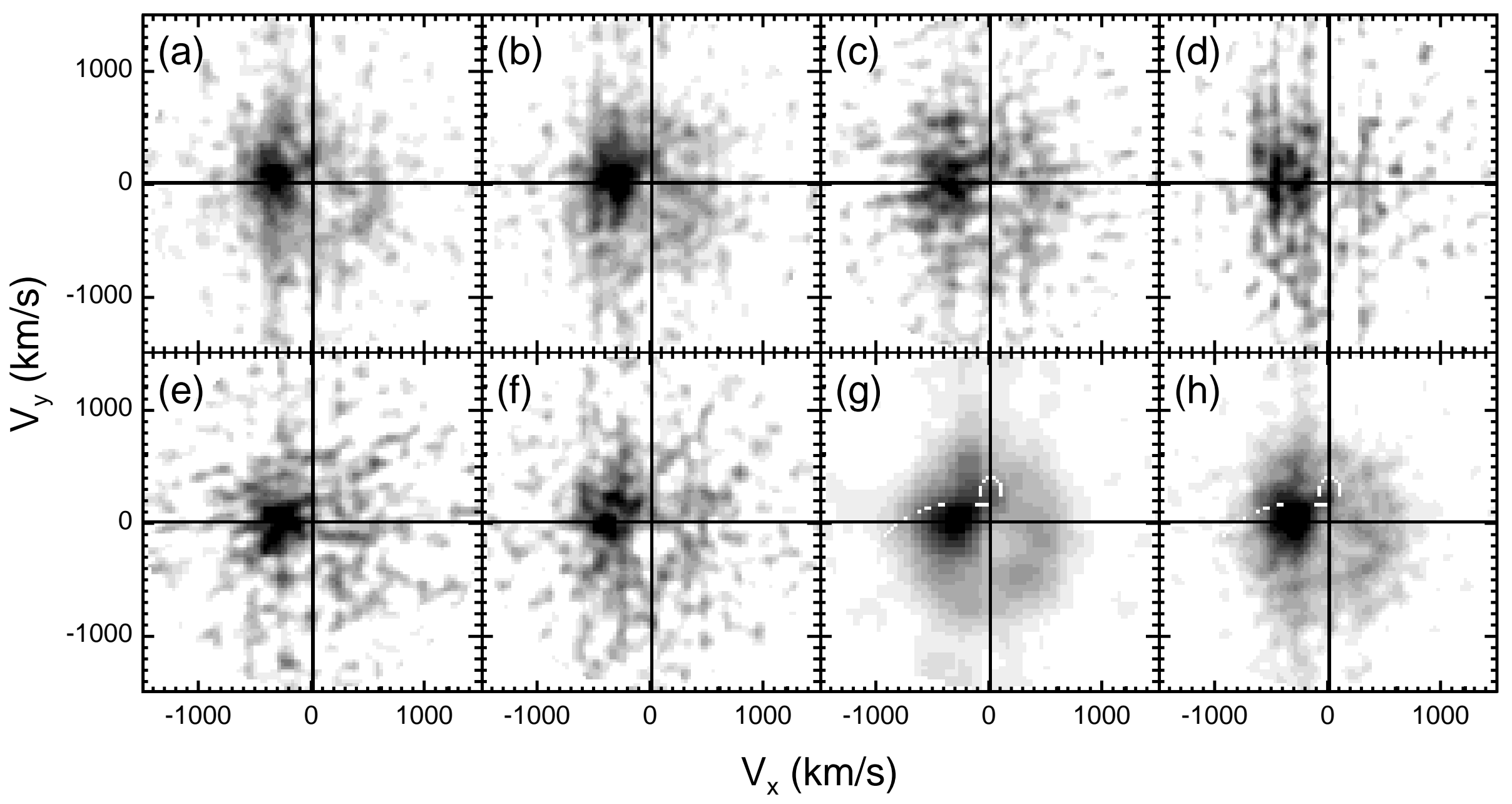




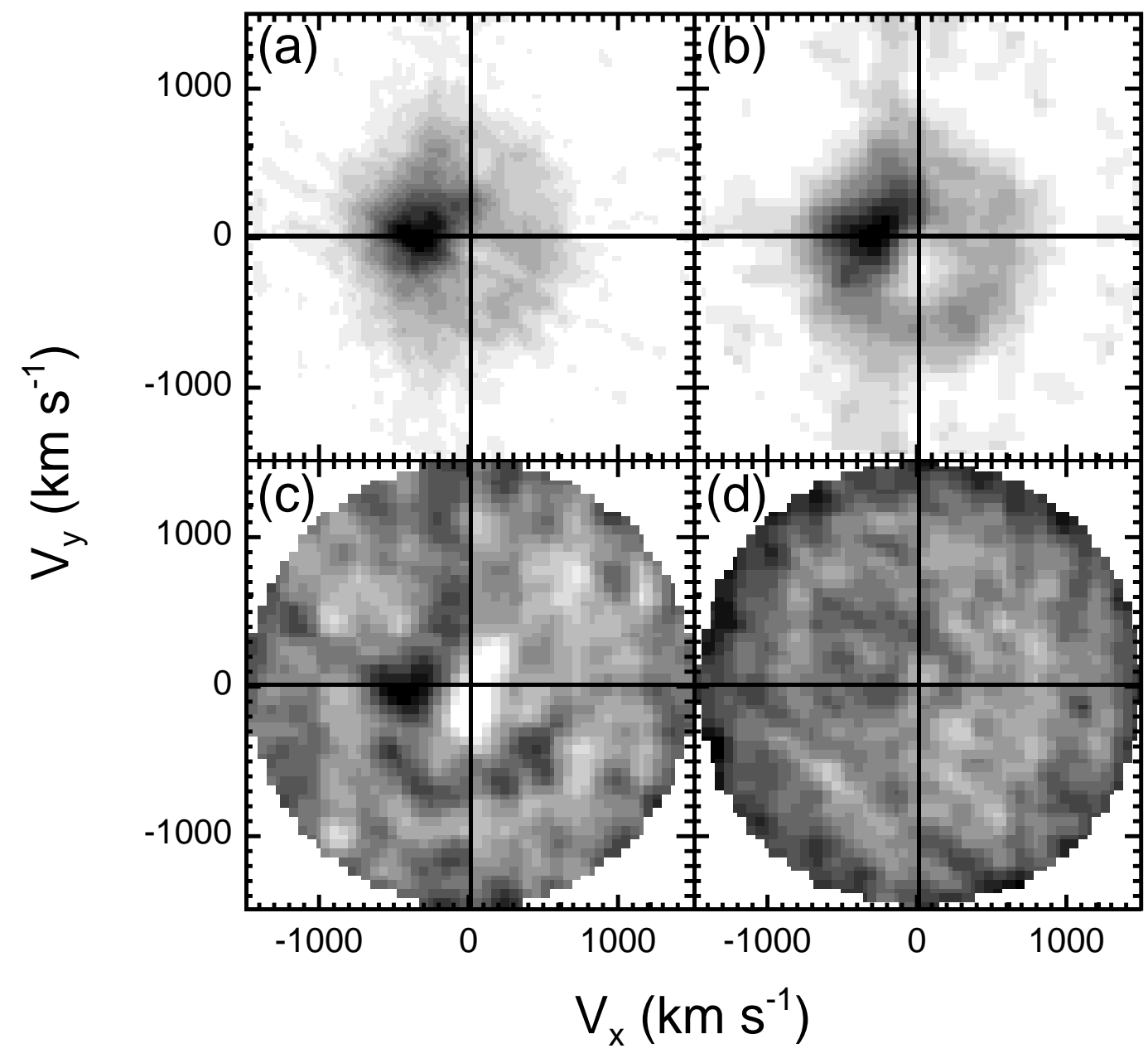




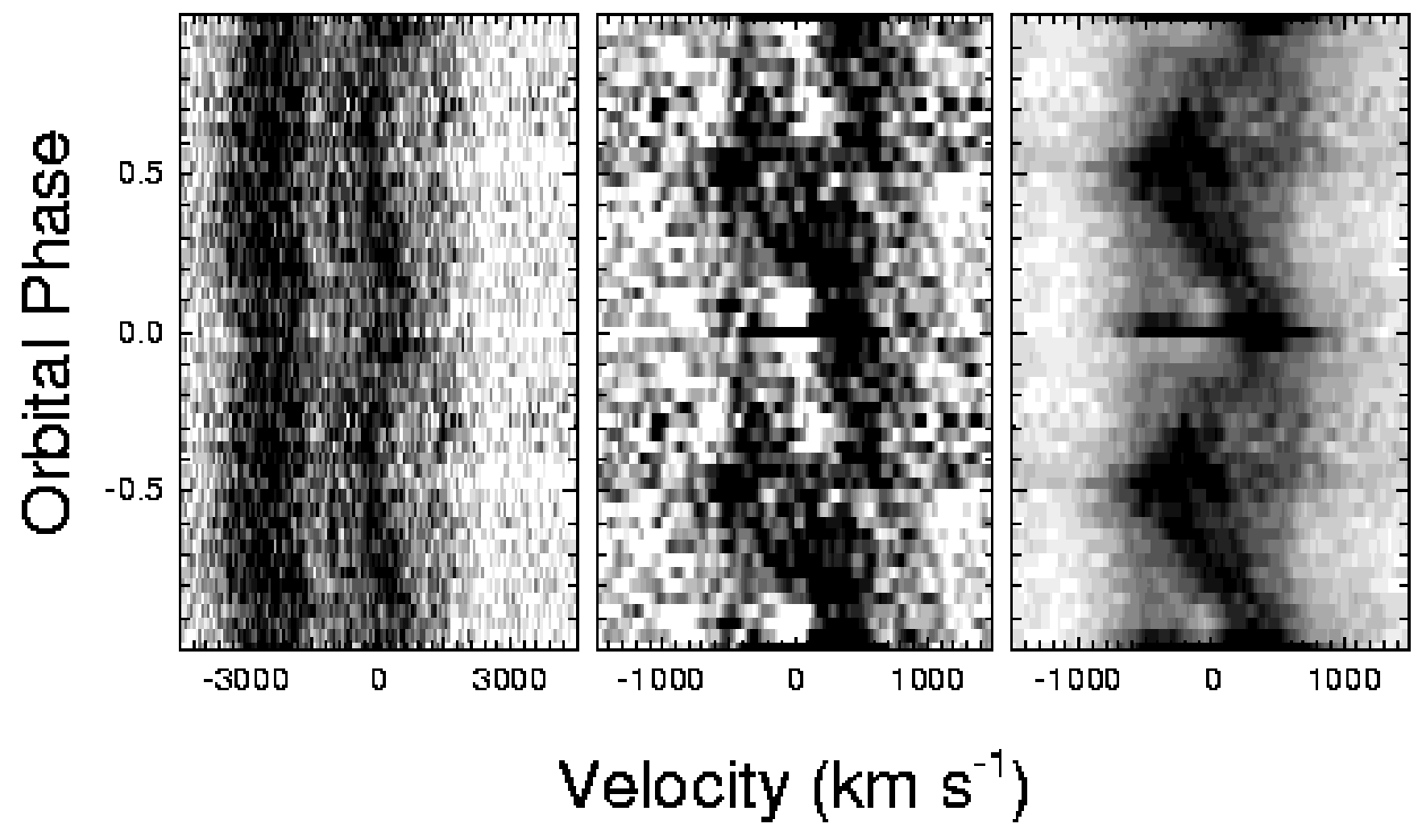




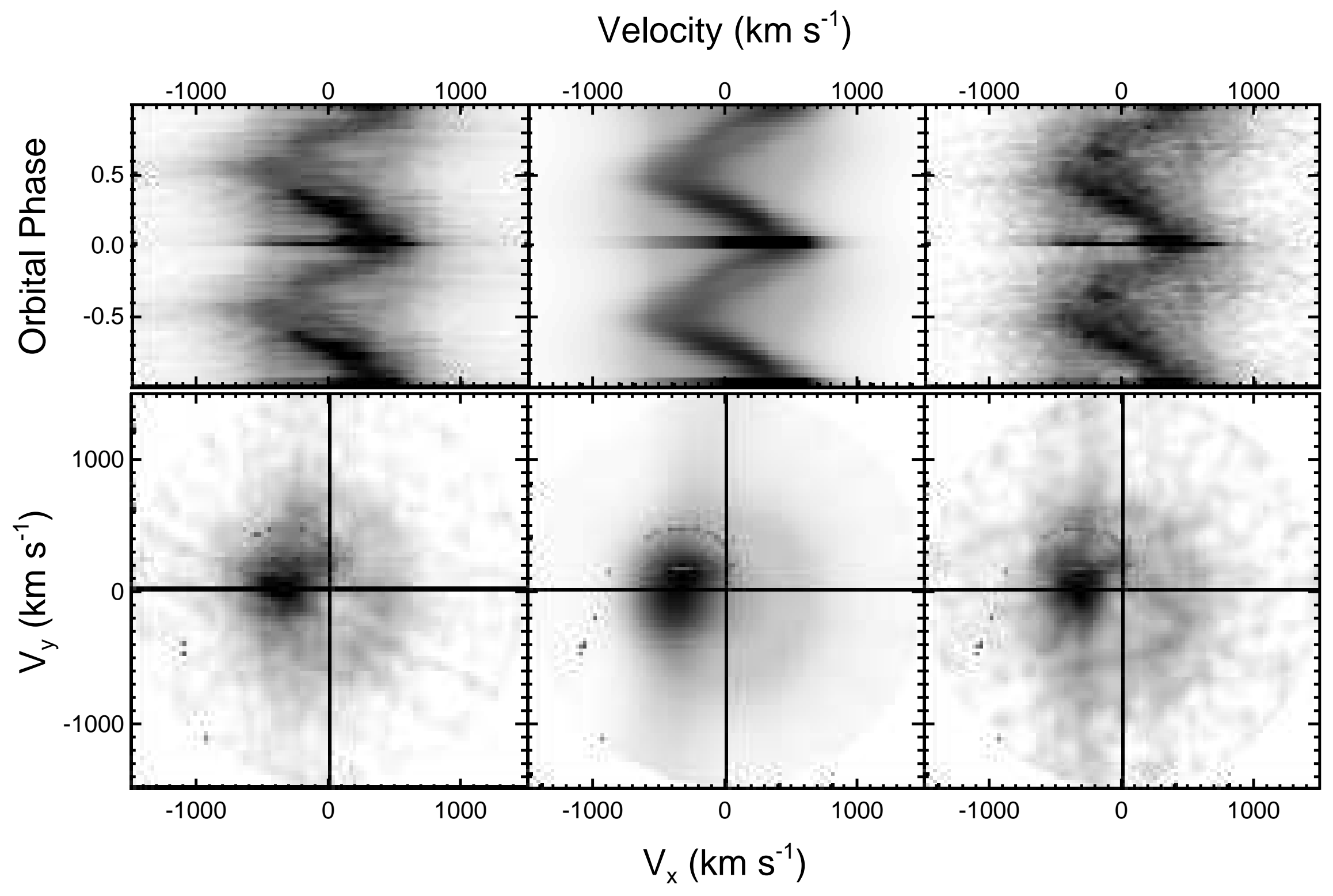



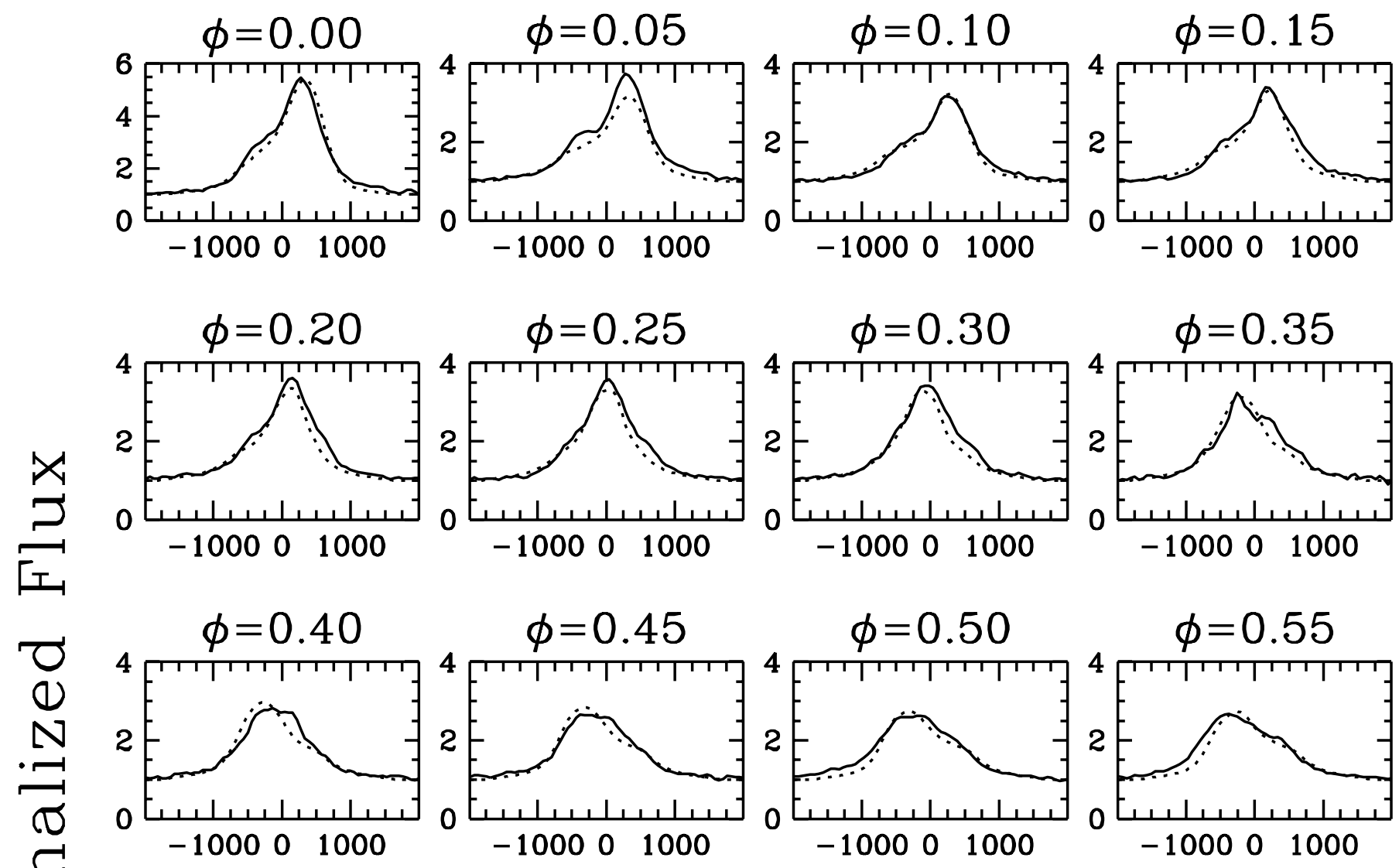

릴
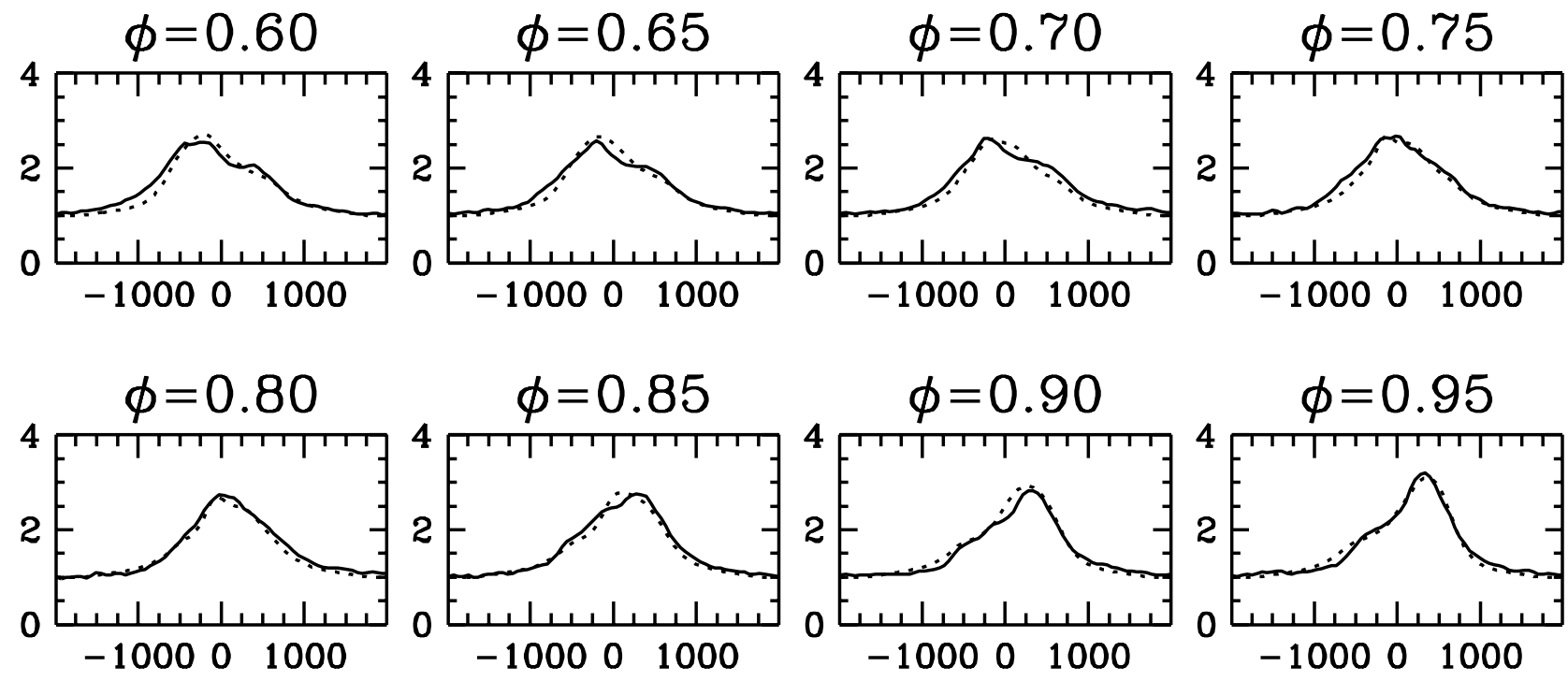

Velocity $\left(\mathrm{km} \mathrm{s}^{-1}\right)$ 


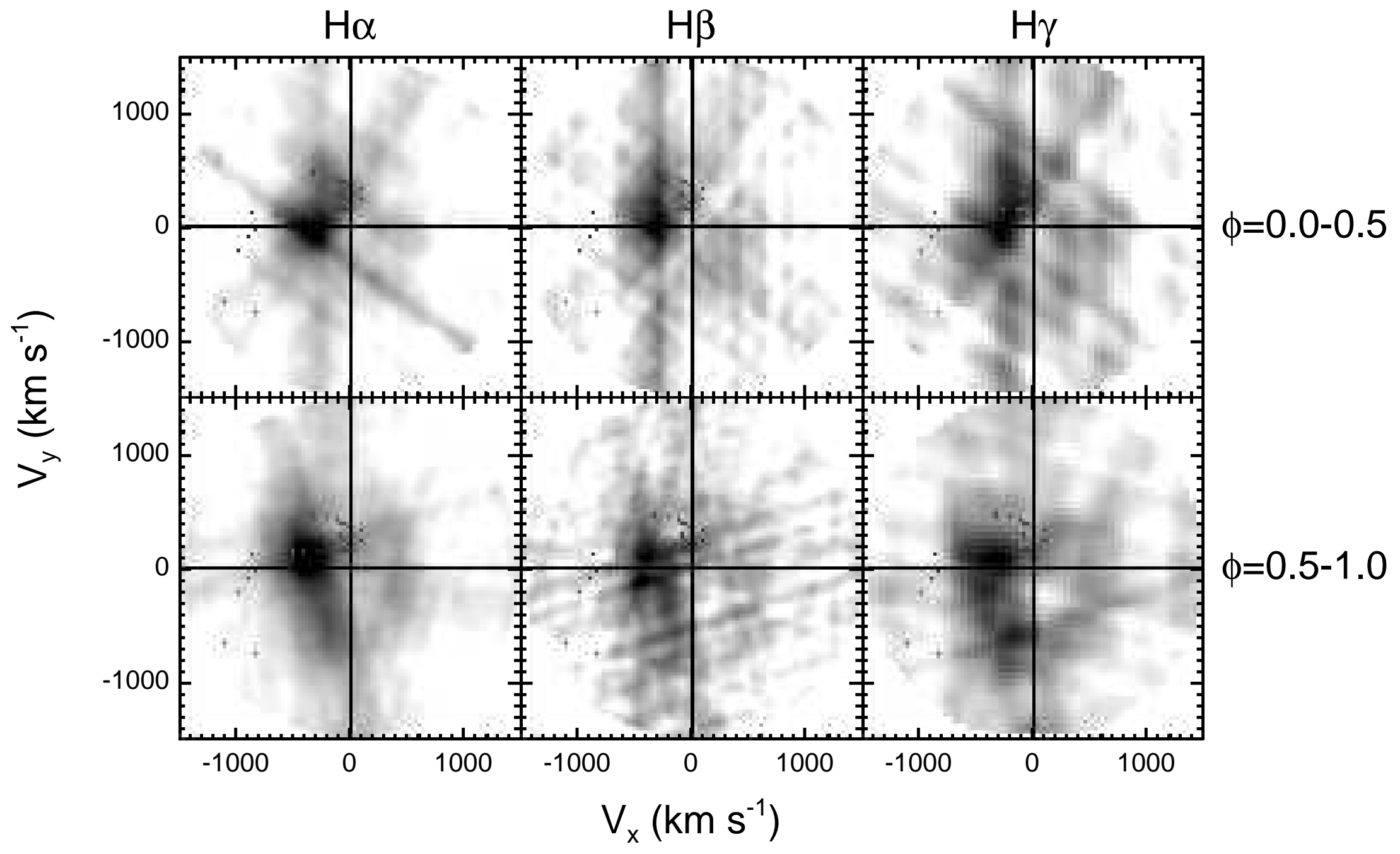




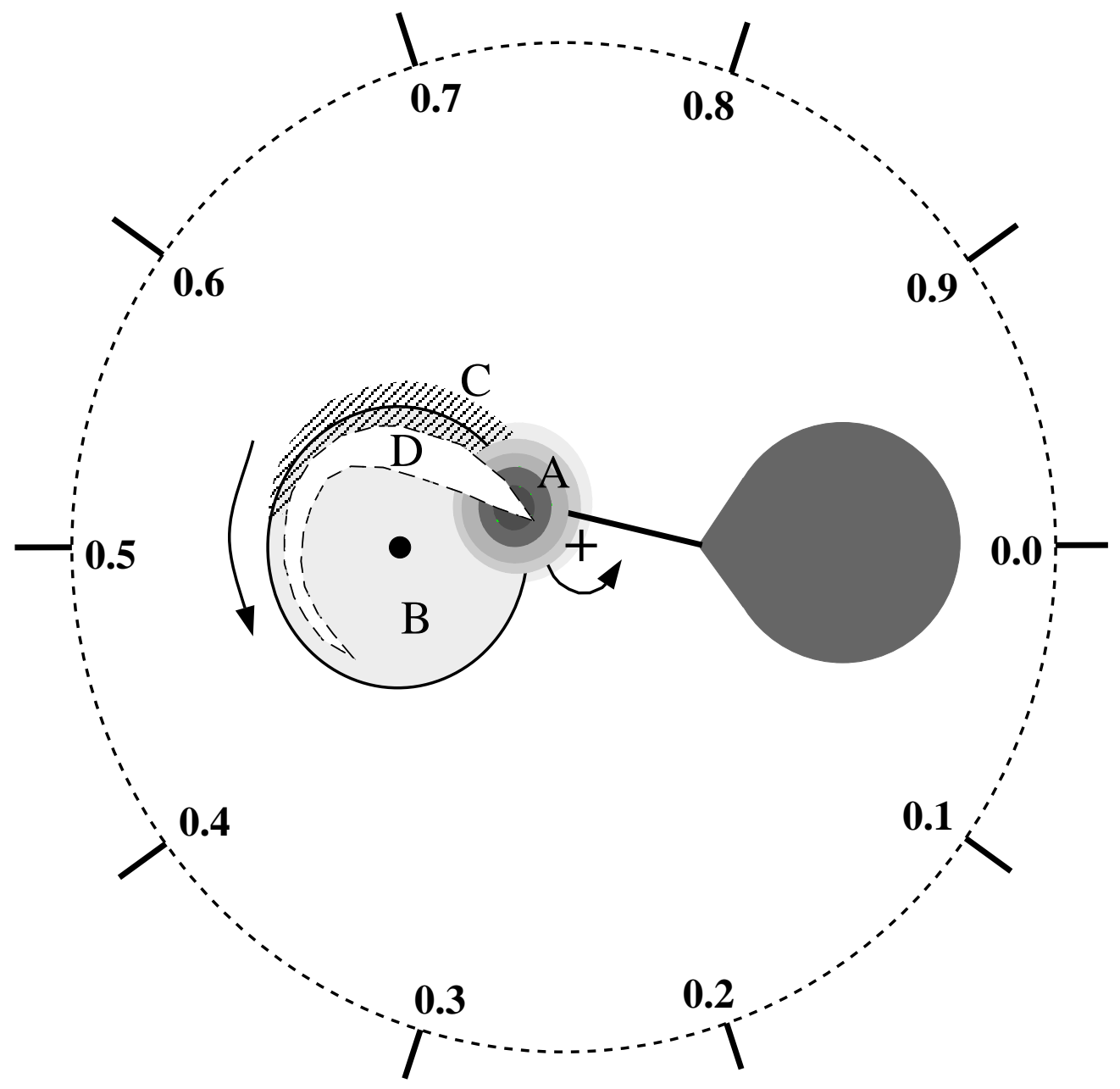

